\title{
COSMOLOGY WITHOUT EINSTEIN'S ASSUMPTION THAT INERTIAL MASS PRODUCES GRAVITY
}

\author{
HOMER G. ELLIS \\ Department of Mathematics, \\ University Colorado Boulder, 395 UCB, \\ Boulder, Colorado 80309-0395, United States of America \\ ellis@euclid.colorado.edu
}

(Dated: May 2, 2015)

\begin{abstract}
Giving up Einstein's assumption, implicit in his 1916 field equations, that inertial mass, even in its appearance as energy, is equivalent to active gravitational mass and therefore is a source of gravity allows revising the field equations to a form in which a positive cosmological constant is seen to (mis)represent a uniform negative net mass density of gravitationally attractive and gravitationally repulsive matter. Field equations with both positive and negative active gravitational mass densities of both primordial and continuously created matter incorporated, along with two scalar fields to 'relax the constraints' on the space-time geometry, yield cosmological solutions that exhibit inflation, deceleration, coasting, acceleration, and a 'big bounce' instead of a 'big bang', and provide good fits to a Hubble diagram of type Ia supernovae data. The repulsive matter is identified as the back sides of the 'drainholes' introduced by the author in 1973 as solutions of those same field equations. Drainholes (prototypical examples of 'traversable wormholes') are topological tunnels in space which gravitationally attract on their front, entrance sides and repel more strongly on their back, exit sides. The front sides serve both as the gravitating cores of the visible, baryonic particles of primordial matter and as the continuously created, invisible particles of the 'dark matter' needed to hold together the large scale structures seen in the universe; the back sides serve as the misnamed 'dark energy' driving the current acceleration of the expansion of the universe. Formation of cosmic voids, walls, filaments, and nodes is attributed to expulsion of drainhole entrances from regions populated by drainhole exits, and accumulation of the entrances on boundaries separating those regions.
\end{abstract}

PACS numbers: PACS numbers: 98.80.Jk, 98.80.Cq, 95.35.+d, 95.36.+x

Keywords: Cosmology; inflation; acceleration; active mass; passive mass; traversable wormhole.

\section{CONTENTS}

I. Introduction

II. Einstein's Implicit Assumption

III. New, Improved Field Equations

IV. Cosmic Evolution Equations

V. Cosmological Solutions

A. Flat open universe $(k=0)$

B. Nonflat open $(k=-1)$ and closed $(k=1)$ universes

VI. Dark Matter and Dark 'Energy'

A. Drainholes

B. Dark matter and dark 'energy' from drainholes

VII. Issues and Observations

A. Gravity and passive-inertial mass

B. Choice of a variational principle for gravity

C. Inflation and the 'big bounce'

D. Dark matter, dark 'energy', and the 'Cosmological Constant Problem'

E. Ratio of dark matter to baryonic matter

F. Continuous creation of C-matter tunnels

G. Voids, walls, filaments, and nodes

H. Protons, neutrons, and WIMPs as drainholes

I. Galactic nuclei as drainholes 


\begin{abstract}
It can scarcely be denied that the supreme goal of all theory is to make the irreducible basic elements as simple and as few as possible without having to surrender the adequate representation of a single datum of experience.

The beginning of wisdom is to call things by their proper name.

\author{
Chinese Proverb (after Confucius)
}

\title{
I. INTRODUCTION
}

In an article published in Journal of Mathematical Physics in 1973 I derived and analyzed in detail a model for a gravitating particle that was an improvement on the Schwarzschild blackhole model [1]. This static and spherically symmetric space-time manifold, discovered independently at about the same time by K. A. Bronnikov [2] and formally some years earlier by O. Bergmann and R. Leipnik who rejected it for "physical reasons" [3], I termed a 'drainhole' with 'ether' flowing through it. The manifold is geodesically complete, singularity-free, and devoid of horizons. It comprises a topological hole (the 'drainhole') connecting two spatial regions, an 'upper' and a 'lower', on which there is a space-time vector field (the 'ether-flow' vector field) representing the velocities of test particles free-falling from rest at infinity in the upper region, into and through the hole, and out into the lower region, accelerating downward all the way. The upper region being asymptotic to a Schwarzschild manifold with positive mass parameter $m$, the lower region is asymptotic to a Schwarzschild manifold with negative mass parameter $\bar{m}=-m e^{m \pi / \sqrt{n^{2}-m^{2}}}$, where $n$ is a parameter that determines the size of the hole. Thus the drainhole attracts test particles on its high side, but repels them on the low side more strongly, in the ratio $-\bar{m} / m=e^{m \pi / \sqrt{n^{2}-m^{2}}}>1$. The drainhole can be thought of as a kind of natural accelerator of the 'gravitational ether', drawing it in on the high side and expelling it more forcefully on the low side. To avoid ambiguities associated with the term 'ether' one could say it is space itself that is flowing into and through the drainhole, a substitution that would accord well with Einstein's insight that the concepts of space and of a gravitational ether are essentially interchangeable [4].

In the 1973 paper I wrote that a "speculative extrapolation from the asymmetry between $m$ and $\bar{m}$ is that the universe expands because it contains more negative mass than positive, each half-particle of positive mass $m$ being slightly overbalanced by a half-particle of negative mass $\bar{m}$ such that $-\bar{m}>m$." This speculation lay dormant until the beginning of 2006, when it occurred to me that the same mechanism might be used to explain not only the expansion of the universe but also the recently discovered acceleration of that expansion. To properly exploit that idea I have found it necessary to reject three of the assumptions that have been built into standard relativistic cosmological theory from its earliest days. The first is Einstein's implicit assumption that active gravitational mass and passive-inertial mass are the same thing, consequently that passive-inertial mass is a source of gravity. The second, which uncritical acceptance of the first gives rise to, is that if a field thought to be associated with some form of matter couples to geometry in the field equations of space-time with the 'wrong' polarity (the 'wrong sign' of the coupling constant), then that form of matter is a 'phantom' or 'ghost' form that can exist only in 'exotic' circumstances. The third is that every scalar field included in a variational principle of relativistic gravitational theory must represent some form of matter and must therefore have its own separate field equation produced by variation of that field in the action integral of the variational principle.

As I shall show, denial of these three assumptions is logically consistent and allows one to arrive at a purely geometric theory of gravitation that produces a singularity-free cosmological model of the universe that fits with very good precision Hubble diagram data from observations of type Ia supernovae. This model features a 'big bounce' (instead of a 'big bang'), rapid inflation out of the bounce, and a 'graceful exit' from the inflation into a long period of decelerative coasting, followed by a transition to an ultimately exponential, de Sitter-like accelerating expansion. In addition, attributing the accelerating expansion to the existence of drainholes provides explanations for dark matter, dark 'energy', and the formation of cosmic voids, walls, filaments, and nodes. The model is offered as a replacement for and improvement on the standard $\Lambda \mathrm{CDM}$ cosmological model. 


\section{EINSTEIN'S IMPLICIT ASSUMPTION}

In his 1916 paper Die Grundlage der allgemeinen Relativitätstheorie [5] that gave a thorough presentation of the theory of gravity he had worked out over the preceding decade, Albert Einstein made implicitly an assumption that does not hold up well under scrutiny. Stripped down to its barest form the assumption is that inertial mass, and by extension energy via $E=m c^{2}$, is a source of gravity and must therefore be coupled to the gravitational potential in the field equations of the general theory of relativity. The train of thought that brought him to this conclusion is described in $\S 16$, where he sought to extend his field equations for the vacuum, $\boldsymbol{R}_{\alpha \beta}-\frac{1}{2} \boldsymbol{R} g_{\alpha \beta}=0$ as currently formulated, to include the contribution of a continuous distribution of gravitating matter of density $\rho$, in analogy to the extension of the Laplace equation $\nabla^{2} \phi=0$ for the newtonian gravitational potential $\phi$ to the Poisson equation $\nabla^{2} \phi=4 \pi \kappa \rho$, where $\kappa$ is Newton's gravitational constant. Einstein referred to $\rho$ as the "density of matter", without specifying what was meant by 'matter' or its 'density'. Invoking the special theory's identification of "inert mass" with "energy, which finds its complete mathematical expression in ... the energy-tensor", he concluded that "we must introduce a corresponding energy-tensor of matter $\mathrm{T}_{\sigma}^{\alpha}$ ". Further describing this energy-tensor as "corresponding to the density $\rho$ in Poisson's equation", he arrived in $\S 19$ at the extended field equations $\boldsymbol{R}_{\alpha \beta}-\frac{1}{2} \boldsymbol{R} g_{\alpha \beta}=\frac{8 \pi \kappa}{c^{2}} T_{\alpha \beta}$, in which, for a "frictionless adiabatic fluid" of "density" $\rho$, "pressure" $p$ (a form of kinetic energy), and proper four-velocity distribution $u^{\alpha}$, he took $T^{\alpha \beta}$ to be $\rho u^{\alpha} u^{\beta}-p g^{\alpha \beta}$ (revised to $\left(\rho+p / c^{2}\right) u^{\alpha} u^{\beta}-p g^{\alpha \beta}$ by his redefinition $\rho \rightarrow \rho+p / c^{2}$ ).

Clearly, Einstein's procedure fails to distinguish between the 'active gravitational mass' of matter, which measures how much gravity it produces and is the sole contributor to the "density of matter" in Poisson's equation, and the "inert mass" of matter, which measures how much it accelerates in response to forces applied to it, an effect entirely different from the production of gravity. These two conceptually different masses, along with yet a third, all occur in Newton's gravitational equation

$$
m_{\text {inertial }} a_{\mathrm{B}}=F_{\mathrm{AB}}=-\kappa \frac{m_{\text {passive }} M_{\text {active }}}{r^{2}},
$$

in which $M_{\text {active }}$ is the active gravitational mass of a gravitating body A, $m_{\text {inertial }}$ is the inertial ("inert") mass of a body $\mathrm{B}$ whose acceleration $a_{\mathrm{B}}$ is caused by the gravity produced by $\mathrm{A}$, and $m_{\text {passive }}$ is the passive gravitational mass of B, a measure of the strength of B's 'sensing' of the gravitational field around A. That in suitable units $m_{\text {inertial }}=m_{\text {passive }}$ for all bodies is another way of saying that all bodies respond with the same accelerations to the same gravitational fields, that, in consequence, the notion of a gravitational field is more fundamental than the notion of a gravitational force. Simple thought experiments of Galileo (large stone and smaller stone tied together) [6] and Einstein (body suspended by a rope in an elevator) [7] make it clear that bodies do all respond alike - an observation now treated as a principle, the (weak) 'principle of equivalence', experimentally, if somewhat redundantly, well confirmed.

That this passive-inertial mass $m_{\text {passive-inertial }}\left(=m_{\text {passive }}=m_{\text {inertial }}\right)$ has any relation to active gravitational mass is not apparent in Eq. (11), where, unlike $m_{\text {inertial }}$ and $m_{\text {passive, }}, M_{\text {active }}$ quantifies a property of A, not of B. But Newton's equation for the gravitational action of B on A reads

$$
M_{\text {inertial }} a_{\mathrm{A}}=F_{\mathrm{BA}}=-\kappa \frac{M_{\text {passive }} m_{\text {active }}}{r^{2}} .
$$

Application of Newton's law of action and reaction allows the inference that $F_{\mathrm{AB}}$ and $F_{\mathrm{BA}}$ have the same magnitude, from which follows that $m_{\text {active }} / m_{\text {passive }}=M_{\text {active }} / M_{\text {passive }}$, hence that the ratio of active gravitational mass to passive gravitational mass, thus to inertial mass, is the same for all bodies. It would seem likely that Einstein relied, either consciously or unconsciously, on this consequence of Newton's laws when he assumed that "inert mass" should contribute to the "density of matter" as a source of gravity in the field equations.

Newton's law of action and reaction is applicable to the bodies A and B only under the condition that gravity acts at a distance instantaneously, that is, at infinite propagation speed. But the general theory of relativity Einstein was expounding is a field theory in which gravitational effects propagate at finite speed. Within his own theory of gravity there is, therefore, no obvious justification for Einstein's assumption that inertial mass (and therefore energy) is equivalent to active gravitational mass. Indeed, in Newton's and in Einstein's theory of gravity in the vacuum the concept of inertial mass is irrelevant, if not in fact nonexistent. In Newton's case cancellation of $m_{\text {inertial }}$ with $m_{\text {passive }}$ from Eq. (11) leaves only $M_{\text {active }}$ to determine the gravitational influence of A on B, namely, an acceleration independent of any property of B. In Einstein's case the same equivalence principle cancellation is effected by his stipulation that test particles in empty space follow the geodesics of the gravitational field as described by the space-time geometry there. That this stipulation leaves in place only the active gravitational mass concept is amply illustrated by the Schwarzschild solution $(1-2 M / r) c^{2} d T^{2}-(1-2 M / r)^{-1} d r^{2}-r^{2} d \Omega^{2}$ of the vacuum field equations $\boldsymbol{R}_{\alpha \beta}-\frac{1}{2} \boldsymbol{R} g_{\alpha \beta}=0$, in which the only mass parameter to appear is the $M$ that determines the curvatures of the space-time metric, thus the strength of the gravitational field of the point particle located at $r=0$. 
Einstein's not recognizing that the inference of equivalence between the concepts of inertial mass and active mass was based on a false premise, then using that inferred equivalence as justification for extending his vacuum field equations to the nonvacuum case in the way he did, was a fundamental error. Few would fault him for such an error made in the midst of the intense intellectual endeavor to construct his general theory of relativity. One can speculate that had he avoided the error he might have discovered in the years after 1916 a number of important things that others discovered only many years later [8].

The failure of Einstein's attempt to introduce inertial mass into his theory by way of his energy-tensor of matter does not preclude the possibility that the concept can be introduced there by some other means. There are in fact good reasons to believe that it should be introduced (or discovered) there as a facet of the space-time geometry, related in some way to the active mass concept. There is, for example, the seemingly universal coincidence that, in physics beyond the merely gravitational, wherever there is matter made of atoms there are to be found both inertial mass and active gravitational mass. Indeed, the fact that Newton's theory gives results that describe as well as they do the motions of the planets and their satellites would argue for some proportionality between $m_{\text {active }}$ and $m_{\text {passive }}$ for such matter in bulk - not, however, for each individual constituent of such matter. A 1986 analysis of lunar ranging data concluded that the ratio of $m_{\text {active }}$ to $m_{\text {passive }}$ for aluminum differs from that for iron by less than $4 \times 10^{-12}$ [9]. An earlier, Cavendish-balance experiment had put a limit of $5 \times 10^{-5}$ on the difference of these ratios for bromine and fluorine [10]. But those results are only for matter in bulk, that is, matter made of atoms and molecules. It is entirely possible that electrons, for example, do not gravitate at all, for no one has ever established by direct observation that they do, nor is it likely that anyone will soon do so. There is in the literature an argument that purports to show that if the ratio $m_{\text {active }} / m_{\text {passive }}$ is the same for two species of bulk matter, then electrons must be generators of gravity [11], but that argument can be seen on careful examination to rest on an unrecognized, hidden assumption of its own, namely that, in simplest form, the gravitational field of a hydrogen atom at a distance could be distinguished from that of a neutron at the same distance [12] - another assumption no one has tested, or is likely soon to test, by direct observation.

Einstein's assumption that energy and inertial mass are sources of gravity has survived to the present virtually unchallenged 1 It has generated a number of consequences that have directed much of the subsequent research in gravitation theory — indeed, misdirected it if his assumption is wrong. Among them are these:

- The impossibility, according to Penrose-Hawking singularity theorems, of avoiding singularities in the geometry of space-time without invoking 'negative energy', which is really just energy coupled to gravity with polarity ('sign') opposite to that of the coupling of matter to gravity.

- The presumption that the extra, fifth dimension in Kaluza-Klein theory must be a spatial dimension rather than a dimension of another type.

- The belief that all the extra dimensions in higher-dimensional theories must be spatial, causing the expenditure of much effort in mental gymnastics to explain why they are not apparent to our senses in the way that the familiar three spatial dimensions are.

Denying Einstein's assumption relieves one of the burden of these troublesome conclusions and opens the door to other, more realistic ones.

\section{NEW, IMPROVED FIELD EQUATIONS}

If Einstein's assumption is to be disallowed, then his source tensor for a continuous distribution of gravitating matter, $T^{\alpha \beta}=\left(\rho+p / c^{2}\right) u^{\alpha} u^{\beta}-p g^{\alpha \beta}$, must be modified or replaced. One might think simply to drop the pressure terms and take $T^{\alpha \beta}=\rho u^{\alpha} u^{\beta}$, the energy-momentum tensor of the matter. This would be inconsistent, for the $\rho$ in that tensor is the density of inertial mass, which we are now not assuming to be the same as active gravitational mass. What should one do instead?

At the same time that Einstein was creating his field equations David Hilbert was deriving the field equations for (in particular) empty space from the variational principle $\delta \int \boldsymbol{R}|g|^{\frac{1}{2}} d^{4} x=0[14]$. This is the most straightforward extension to the general relativity setting of the variational principle $\delta \int|\nabla \phi|^{2} d^{3} x=0$, whose Euler-Lagrange equation is equivalent to the Laplace equation $\nabla^{2} \phi=0$ for the newtonian potential $\phi$. Modifying that principle to $\delta \int\left(|\nabla \phi|^{2}+\right.$ $8 \pi \kappa \mu \phi) d^{3} x=0$, where $\mu$ is the density of the active gravitational mass of matter, generates the Poisson equation

${ }^{1}$ Curiously, Herman Bondi in a paper in 1957 carefully distinguished between passive-inertial mass and active mass, then in the same paper adopted Einstein's "energy-tensor" which ignores the distinction [13]. 
$\nabla^{2} \phi=4 \pi \kappa \mu$. The most straightforward extension of this modified principle to general relativity is

$$
\delta \int\left(\boldsymbol{R}-\frac{8 \pi \kappa}{c^{2}} \mu\right)|g|^{\frac{1}{2}} d^{4} x=0,
$$

for which the Euler-Lagrange equations are equivalent to

$$
\boldsymbol{R}_{\alpha \beta}-\frac{1}{2} \boldsymbol{R} g_{\alpha \beta}=-\frac{4 \pi \kappa}{c^{2}} \mu g_{\alpha \beta},
$$

which makes $T_{\alpha \beta}=-\frac{1}{2} \mu g_{\alpha \beta}$. Equivalent to this equation is $\boldsymbol{R}_{\alpha \beta}=\frac{4 \pi \kappa}{c^{2}} \mu g_{\alpha \beta}$, the 00 component of which reduces in the slowly varying, weak field approximation to the Poisson equation, with $\phi=\frac{1}{2}\left(g_{00}-c^{2}\right)$.

The vanishing of the divergence of the Einstein tensor field $\boldsymbol{R}_{\alpha \beta}-\frac{1}{2} \boldsymbol{R} g_{\alpha \beta}$ in Eq. (4) entails that $0=T_{\alpha}{ }^{\beta}: \beta=$ $-\frac{1}{2}\left(\mu_{. \beta} g_{\alpha}{ }^{\beta}+\mu g_{\alpha}{ }^{\beta}: \beta\right)=-\frac{1}{2} \mu_{. \alpha}$, hence that $\mu$ is both spatially and temporally constant. These constraints on $\mu$ being unduly restrictive, further modification is in order.

To widen the range of space-time geometries admitted by the field equations one can in the usual way add to the action integrand of Eq. (3) terms related to such things as scalar fields and electromagnetic fields. In particular, one can add a cosmological constant term, changing the integrand to $\boldsymbol{R}-\frac{8 \pi \kappa}{c^{2}} \mu+2 \Lambda$ and the field equations to

$$
\boldsymbol{R}_{\alpha \beta}-\frac{1}{2} \boldsymbol{R} g_{\alpha \beta}=-\frac{4 \pi \kappa}{c^{2}} \mu g_{\alpha \beta}+\Lambda g_{\alpha \beta}=-\frac{4 \pi \kappa}{c^{2}}(\mu+\bar{\mu}) g_{\alpha \beta},
$$

where $\bar{\mu}=-\frac{c^{2}}{4 \pi \kappa} \Lambda$. A positive cosmological constant $\Lambda$ thus appears in this context to be a (mis)representation of a negative active mass density $\bar{\mu}$ of a continuous distribution of gravitationally repulsive matter. The same field equations are obtained by changing the integrand to $\boldsymbol{R}+2 \Lambda$ and setting $\Lambda=-\frac{4 \pi \kappa}{c^{2}}(\mu+\bar{\mu})$, thus associating a positive $\Lambda$ with a negative net active mass density of gravitating matter, some attractive, some repulsive. As suggested in the Introduction, an excess of the negative active mass density $\bar{\mu}$ of repulsive matter over the positive density $\mu$ of attractive matter could drive an accelerating cosmic expansion (and in the process would solve the vexing 'Cosmological Constant Problem' by identifying $-\frac{c^{2}}{4 \pi \kappa} \Lambda$ as the net density $\mu+\bar{\mu}$ of gravitating matter). Leaving for later a full discussion of drainholes as the source of such a density imbalance (cf. Sec. VIA), we can explore the consequences of an imbalance by studying cosmological solutions of field equations that incorporate a positive mass density $\mu$, a negative mass density $\bar{\mu}$, and scalar fields $\phi$ (not the newtonian $\phi$ ) and $\psi$, all folded into the space-time geometry via the variational principle

$$
\delta \int\left[\boldsymbol{R}-\frac{8 \pi \kappa}{c^{2}}(\mu+\bar{\mu})+2 \phi^{\cdot \gamma} \phi_{\cdot \gamma}-2 \psi^{\cdot \gamma} \psi_{\cdot \gamma}\right]|g|^{\frac{1}{2}} d^{4} x=0 .
$$

In deriving field equations from this variational principle I will vary only the space-time metric. Not varying the densities is normal, but not varying the scalar fields goes against orthodox practice. The rationale for leaving them unvaried is this: In a space-time manifold the geometry is determined by the metric alone, so only the metric should participate in the extremizing of the action; to vary the scalar fields would be to treat them as something extraneous to the metric thus to the geometry, whereas their proper role should be simply to introduce a useful flexibility into the extremizing process, not to represent explicit contributions to gravity by the 'energy' fields of distributions of scalar matter. Varying neither the scalar fields nor the densities, but only the metric, is in keeping with Einstein's guiding principle that geometry should be able to explain all of physics.

Looked at another way, including the terms $-\frac{8 \pi \kappa}{c^{2}} \mu,-\frac{8 \pi \kappa}{c^{2}} \bar{\mu}, 2 \phi^{\cdot \gamma} \phi_{. \gamma}$, and $-2 \psi^{\cdot \gamma} \psi_{. \gamma}$ in the action can be seen as allowing 'relaxations' of the metric, analogously to accommodating 'constraints' on the metric by including Lagrange multipliers in the action (e. g., $\lambda$ in the action $\int(\boldsymbol{R}+\lambda)|g|^{\frac{1}{2}} d^{n} x=\int \boldsymbol{R}|g|^{\frac{1}{2}} d^{n} x+\lambda \int|g|^{\frac{1}{2}} d^{n} x$ for an $n$-dimensional Einstein manifold, to accommodate the constraint that the volume $\int|g|^{\frac{1}{2}} d^{n} x$ of the integration region is held fixed). Just as one does not vary Lagrange multipliers, one should not vary $\mu, \bar{\mu}, \phi$, or $\psi$. In this role $\mu, \bar{\mu}, \phi$, and $\psi$ simply relax the field equations to allow a larger class of metrics to satisfy them. The relaxed field equations will be as useful as the metrics that satisfy them, no more, no less.

Breaking the scalar field portion of the action into two parts, one $\left(2 \psi^{\cdot \gamma} \psi_{\cdot \gamma}\right)$ coupled to geometry with the orthodox polarity, the other $\left(2 \phi^{\cdot \gamma} \phi_{. \gamma}\right)$ coupled with the opposite ('phantom' or 'ghost') polarity is justified by the absence of any compelling reason for choosing one coupling over the other. The usual mantra accompanying the making of such a choice is that a scalar field coupled to geometry with the 'wrong sign' has 'negative energy' and therefore represents 'exotic matter' that can exist if at all only in highly contrived circumstances. This misconception traces back to Einstein's mistaking as active the passive-inertial density $\rho$ in his "energy-tensor of matter $\mathrm{T}_{\sigma}^{\alpha}$ ". If his field equations $\boldsymbol{R}_{\alpha \beta}-\frac{1}{2} \boldsymbol{R} g_{\alpha \beta}=\frac{8 \pi \kappa}{c^{2}} T_{\alpha \beta}$ were changed to $\boldsymbol{R}_{\alpha \beta}-\frac{1}{2} \boldsymbol{R} g_{\alpha \beta}=-\frac{8 \pi \kappa}{c^{2}} T_{\alpha \beta}$, the effect, in the absence of pressure, would be equivalent to taking the density $\rho$ to be negative in the original equations, in which case the matter it 
purports to represent would be gravitationally repulsive, and 'exotic' for having negative inertial mass and therefore negative energy. Correcting Einstein's mistake reveals the constructions 'exotic matter with negative energy density', 'phantom field', 'ghost field', and others like them to be little more than instances of misleading jargon.

Variation of the metric in the action integral of Eq. (6) generates the field equations

$$
\boldsymbol{R}_{\alpha \beta}-\frac{1}{2} \boldsymbol{R} g_{\alpha \beta}=\frac{8 \pi \kappa}{c^{2}} T_{\alpha \beta}
$$

with

$$
\begin{aligned}
\frac{8 \pi \kappa}{c^{2}} T_{\alpha \beta}:=-\frac{4 \pi \kappa}{c^{2}}(\mu+\bar{\mu}) g_{\alpha \beta} & -2\left(\phi_{. \alpha} \phi_{. \beta}-\frac{1}{2} \phi^{\cdot \gamma} \phi_{. \gamma} g_{\alpha \beta}\right) \\
& +2\left(\psi_{. \alpha} \psi_{. \beta}-\frac{1}{2} \psi^{\cdot \gamma} \psi_{. \gamma} g_{\alpha \beta}\right),
\end{aligned}
$$

and, in consequence,

$$
2(\square \phi) \phi_{. \alpha}-2(\square \psi) \psi_{. \alpha}:=2 \phi_{: \gamma}^{\cdot \gamma} \phi_{. \alpha}-2 \psi^{\cdot \gamma}{ }_{: \gamma} \psi_{. \alpha}=-\frac{4 \pi \kappa}{c^{2}}(\mu+\bar{\mu})_{. \alpha} .
$$

The latter of these, which follows from the vanishing of the divergence of $T_{\alpha \beta}$ implied by the canonical (Bianchi identity) vanishing of the divergence of $\boldsymbol{R}_{\alpha \beta}-\frac{1}{2} \boldsymbol{R} g_{\alpha \beta}$ in the former, is what one has in place of the wave equations $\square \phi=0$ and $\square \psi=0$ that would have resulted from varying $\phi$ and $\psi$. It leaves $\phi$ and $\psi$ underdetermined, which is consistent with their roles as 'relaxants' to allow a wider range of metrics to satisfy the field equations than would be allowed in their absence.

The next two sections will test the usefulness of these field equations by examining a homogeneous cosmological model they admit as a solution.

\section{COSMIC EVOLUTION EQUATIONS}

For a Robertson-Walker metric $c^{2} d t^{2}-R^{2}(t) d s^{2}$ (with $t$ in seconds, $s$ dimensionless, $c$ in meters per second, and $R$ in meters) and dimensionless scalar fields $\phi=\alpha(t)$ and $\psi=\beta(t)$ the field Eqs. (7) reduce to

$$
3 \frac{\dot{R}^{2} / c^{2}+k}{R^{2}}=-\frac{4 \pi \kappa}{c^{2}}(\mu+\bar{\mu})-\frac{\dot{\alpha}^{2}-\dot{\beta}^{2}}{c^{2}},
$$

and

$$
\frac{2}{c^{2}} \frac{\ddot{R}}{R}+\frac{\dot{R}^{2} / c^{2}+k}{R^{2}}=-\frac{4 \pi \kappa}{c^{2}}(\mu+\bar{\mu})+\frac{\dot{\alpha}^{2}-\dot{\beta}^{2}}{c^{2}},
$$

where $k=-1,0$, or 1 , the uniform curvature of the spatial metric $d s^{2}$. These equations, which are replacements for the well-studied Friedmann-Lemaitre cosmological equations, are equivalent together to

$$
\frac{1}{c^{2}} \frac{\dot{R}^{2}}{R^{2}}=-\frac{4 \pi \kappa}{3 c^{2}}(\mu+\bar{\mu})-\frac{k}{R^{2}}-\frac{\dot{\alpha}^{2}-\dot{\beta}^{2}}{3 c^{2}}
$$

and

$$
\frac{1}{c^{2}} \frac{\ddot{R}}{R}=-\frac{4 \pi \kappa}{3 c^{2}}(\mu+\bar{\mu})+\frac{2\left(\dot{\alpha}^{2}-\dot{\beta}^{2}\right)}{3 c^{2}} .
$$

Equations (9) reduce to

$$
\frac{2}{c^{2}}\left(\ddot{\alpha}+3 \frac{\dot{R}}{R} \dot{\alpha}\right) \dot{\alpha}-\frac{2}{c^{2}}\left(\ddot{\beta}+3 \frac{\dot{R}}{R} \dot{\beta}\right) \dot{\beta}=-\frac{4 \pi \kappa}{c^{2}} \partial_{t}(\mu+\bar{\mu})
$$

for the time component and $\partial_{a}(\mu+\bar{\mu})=0$ for the space components, thus impose spatial but not temporal uniformity on $\mu+\bar{\mu}$. Equation (14) is equivalent to

$$
\frac{1}{c^{2}}\left(R^{6}\left(\dot{\alpha}^{2}-\dot{\beta}^{2}\right)\right) \cdot=-\frac{4 \pi \kappa}{c^{2}} R^{6} \partial_{t}(\mu+\bar{\mu}) .
$$


To give substance to the densities $\mu$ and $\bar{\mu}$ let us invoke, as assumed ingredients of the model under construction, two kinds of gravitating matter: a) primordial matter, existent at all times, never changing in amount, with its net density $\mu+\bar{\mu}$ thus inversely proportional to the cube of the scale factor $R$; and b) continuously created (or destroyed) matter, coming steadily into existence (or passing out of existence) at a rate just sufficient to keep its net density constant. The first of these is, except for the inclusion of a repulsive component of the density, not essentially different from what one assumes for the $\Lambda \mathrm{CDM}$ model. The second is essentially the same as the basic assumption on which the so-called 'steady state' cosmology is built, also excepting the repulsive density component. Incorporating both will allow our model to provide a good fit to relevant astronomical observations.

For the net density of primordial matter we have

$$
\mu_{\mathrm{P}}(t)+\bar{\mu}_{\mathrm{P}}(t)=\left(\mu_{\mathrm{P}, 0}+\bar{\mu}_{\mathrm{P}, 0}\right) \frac{R^{3}\left(t_{0}\right)}{R^{3}(t)},
$$

where $t_{0}$ denotes the value of $t$ at the present epoch, and $\mu_{\mathrm{P}, 0}$ and $\bar{\mu}_{\mathrm{P}, 0}$ are the present values of the primordial densities $\mu_{\mathrm{P}}$ and $\bar{\mu}_{\mathrm{P}}$. For the net density of continuously created matter we have

$$
\mu_{\mathrm{C}}(t)+\bar{\mu}_{\mathrm{C}}(t)=\mu_{\mathrm{C}, 0}+\bar{\mu}_{\mathrm{C}, 0},
$$

where $\mu_{\mathrm{C}, 0}$ and $\bar{\mu}_{\mathrm{C}, 0}$ are the present values of the continuously created densities $\mu_{\mathrm{C}}$ and $\bar{\mu}_{\mathrm{C}}$. Under the assumption that the net densities $\mu_{\mathrm{P}}+\bar{\mu}_{\mathrm{P}}$ and $\mu_{\mathrm{C}}+\bar{\mu}_{\mathrm{C}}$ are additive, i. e., $\mu+\bar{\mu}=\left(\mu_{\mathrm{P}}+\bar{\mu}_{\mathrm{P}}\right)+\left(\mu_{\mathrm{C}}+\bar{\mu}_{\mathrm{C}}\right)$, Eq. (15) turns into

$$
\frac{1}{c^{2}}\left(R^{6}\left(\dot{\alpha}^{2}-\dot{\beta}^{2}\right)\right)^{\cdot}=\frac{4 \pi \kappa}{c^{2}}\left(\mu_{\mathrm{P}, 0}+\bar{\mu}_{\mathrm{P}, 0}\right) R^{3}\left(t_{0}\right)\left(R^{3}\right)^{\cdot},
$$

which integrates to

$$
\frac{1}{c^{2}}\left(\dot{\alpha}^{2}-\dot{\beta}^{2}\right)=\frac{4 \pi \kappa}{c^{2}}\left(\mu_{\mathrm{P}, 0}+\bar{\mu}_{\mathrm{P}, 0}\right) \frac{R^{3}\left(t_{0}\right)}{R^{3}}+\frac{B}{R^{6}},
$$

where $B$ is the constant of integration, with units $\mathrm{m}^{4}$.

At this point it is convenient to set

$$
\left.A_{\mathrm{P}}:=-\frac{4 \pi \kappa}{c^{2}}\left(\mu_{\mathrm{P}, 0}+\bar{\mu}_{\mathrm{P}, 0}\right) R^{3}\left(t_{0}\right) \quad \text { (units } \mathrm{m}\right)
$$

and

$$
A_{\mathrm{C}}:=-\frac{4 \pi \kappa}{c^{2}}\left(\mu_{\mathrm{C}, 0}+\bar{\mu}_{\mathrm{C}, 0}\right) \quad\left(\text { units }^{-2}\right) .
$$

Equation (19) then becomes

$$
\frac{1}{c^{2}}\left(\dot{\alpha}^{2}-\dot{\beta}^{2}\right)=\frac{B-A_{\mathrm{P}} R^{3}}{R^{6}},
$$

and substitution from Eqs. (20), (21), and (22) into Eqs. (12) and (13) produces

$$
\frac{1}{c^{2}} \frac{\dot{R}^{2}}{R^{2}}=\frac{P_{1}(R)}{3 R^{6}}=\frac{A_{\mathrm{C}}}{3}-\frac{k}{R^{2}}+\frac{2 A_{\mathrm{P}}}{3 R^{3}}-\frac{B}{3 R^{6}}
$$

and

$$
\frac{1}{c^{2}} \frac{\ddot{R}}{R}=\frac{P_{2}(R)}{3 R^{6}}=\frac{A_{\mathrm{C}}}{3}-\frac{A_{\mathrm{P}}}{3 R^{3}}+\frac{2 B}{3 R^{6}},
$$

where

$$
P_{1}(R):=A_{\mathrm{C}} R^{6}-3 k R^{4}+2 A_{\mathrm{P}} R^{3}-B
$$

and

$$
P_{2}(R):=A_{\mathrm{C}} R^{6}-A_{\mathrm{P}} R^{3}+2 B .
$$

According to Eqs. (20) and (21), positive values for $A_{\mathrm{P}}$ and $A_{\mathrm{C}}$ correspond to negative net densities for primordial matter and continuously created matter, signifying that on balance matter of each kind gravitationally repels all other matter more strongly than it attracts other matter. This excess of repulsion over attraction will be presumed to exist for both types. With thus $A_{\mathrm{P}}>0$ and $A_{\mathrm{C}}>0$ assumed, along with an added assumption that $B>0$, several properties of the scale factor $R$ as a solution of these equations can be inferred rather easily, to wit: 

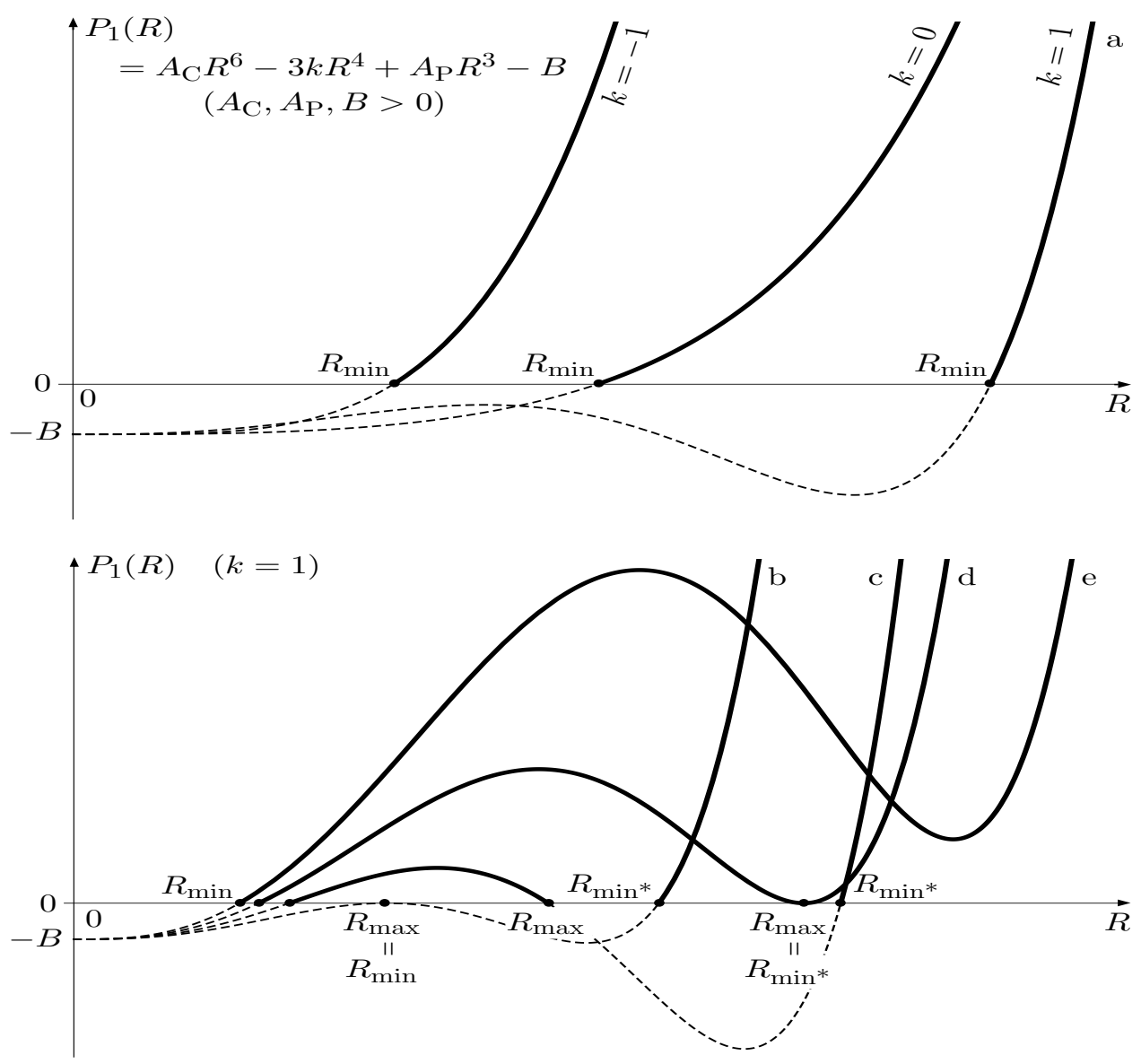

FIG. 1. Graphs of $P_{1}(R)$ for $k=-1,0$, and 1 , and generic positive values of the parameters $A_{\mathrm{C}}, A_{\mathrm{P}}$, and $B$. Values of $R$ for which $P_{1}(R)<0$ are excluded from the range of $R(t)$ by Eq. (23).

- For each of $k=-1,0,1$ there is a least positive number $R_{\min }$ (the least positive root of the polynomial $P_{1}(R)$ ) that $R$ cannot go below without violating Eq. (23), and at which $\dot{R}=0$. (See Fig. (1) This sets a positive lower bound on the compression of space and thereby rules out the development of a 'big bang' singularity.

- For $k=1$ and some choices of $A_{\mathrm{C}}, A_{\mathrm{P}}$, and $B$ (Fig. 1, graphs b, c, and d) there are in addition to $R_{\min }$ other values $R_{\max }$ and $R_{\min } *$ of $R$ at which $P_{1}$, and consequently $\dot{R}$, vanish.

- The identity $P_{2}(R)=\frac{1}{2} R P_{1}^{\prime}(R)-2 P_{1}(R)$ has the consequence that because, with one exception (Fig. 1 graph b), $P_{1}^{\prime}\left(R_{\min }\right)>0$, also $P_{2}\left(R_{\min }\right)>0$, and therefore $\ddot{R}$ is positive when $R=R_{\min }$. This tells that in place of a 'big bang' there is a 'bounce' off a state of maximum compression at the time when $R=R_{\min }$. The lone exception has $k=1, P_{2}\left(R_{\min }\right)=P_{1}^{\prime}\left(R_{\min }\right)=0$, and $R(t)=R_{\min }=R_{\max }$ for all time, modeling a static, spherical universe of radius $R_{\min }$. A similar model (Fig. 1 graph d) has $R(t)=R_{\min ^{*}}=R_{\max }$ for all time, and also as an asymptotic limit from below and from above.

- With the time $t=0$ chosen so that $R(0)=R_{\min }, R(t)$ is symmetric about $t=0$, the evolution of the universe after time zero thus being mirrored in reverse as a devolution of the universe before time zero.

- Whereas for $k=-1$ and $k=0$ (Fig. 1), and $k=1$ (Fig. 1) graph a), the universe expands from $R_{\min }$ to infinity as $t \rightarrow \pm \infty$, other behaviors are possible when $k=1$, most notably: (Fig. 1, graph b) static spherical universe as noted above; (Fig. 1, graph c) spherical universe oscillating for all time between minimum radius $R_{\min }$ and maximum radius $R_{\text {max }}$.

- The polynomial $P_{2}(R)$ is quadratic in $R^{3}$, with discriminant $A_{\mathrm{P}}^{2}-8 A_{\mathrm{C}} B$. If $B>A_{\mathrm{P}}^{2} / 8 A_{\mathrm{C}}$, then $P_{2}(R)$ has no positive root, so $\ddot{R}>0$ at all times, thus the universal expansion described by $R(t)$ for times after $t=0$ is always accelerating. If $B=A_{\mathrm{P}}^{2} / 8 A_{\mathrm{C}}$, then $P_{2}(R)=A_{\mathrm{C}}\left(R^{3}-A_{\mathrm{P}} / 2 A_{\mathrm{C}}\right)^{2}$, so the expansion is, except for a 
momentary pause when $R(t)$ passes through $\sqrt[3]{A_{\mathrm{P}} / 2 A_{\mathrm{C}}}$, accelerating at all positive times, unless, as in Fig. 1. graph b, $R(t)=R_{\min }=R_{\max }$ or, as in Fig. 1 graph d, $R(t)=R_{\text {min* }^{*}}=R_{\max }$. If $B<A_{\mathrm{P}}^{2} / 8 A_{\mathrm{C}}$, then $P_{2}(R)$ has two positive roots,

$$
R_{\mathrm{d}}:=\left(\frac{A_{\mathrm{P}}}{2 A_{\mathrm{C}}}-\frac{\sqrt{A_{\mathrm{P}}^{2}-8 A_{\mathrm{C}} B}}{2 A_{\mathrm{C}}}\right)^{\frac{1}{3}},
$$

which marks a transition from the initial accelerating expansion associated with the bounce at $t=0$ to an interval of decelerating expansion, and

$$
R_{\mathrm{a}}:=\left(\frac{A_{\mathrm{P}}}{2 A_{\mathrm{C}}}+\frac{\sqrt{A_{\mathrm{P}}^{2}-8 A_{\mathrm{C}} B}}{2 A_{\mathrm{C}}}\right)^{\frac{1}{3}},
$$

which marks a return to accelerating expansion (if the expansion is destined to continue forever, as opposed to some of the other possible behaviors when $k=1$ ).

- For the infinitely expanding models the 'Hubble parameter' $H(:=\dot{R} / R)$ and the 'acceleration parameter' $Q$ $\left(:=(\ddot{R} / R) /(\dot{R} / R)^{2}\right)$ behave asymptotically as follows:

$$
\begin{aligned}
\frac{1}{c^{2}} H^{2}=\frac{P 1(R)}{3 R^{6}} & =\frac{A_{\mathrm{C}}}{3}-\frac{3 k R^{4}-2 A_{\mathrm{P}} R^{3}+B}{3 R^{6}} \\
& \rightarrow \frac{A_{\mathrm{C}}}{3} \quad\left\{\begin{array}{l}
\text { from below if } k>0 \\
\text { from above if } k \leq 0
\end{array}\right\} \text { as } R \rightarrow \infty
\end{aligned}
$$

(telling that, for some number $C, R(t) \sim C e^{ \pm \sqrt{A_{\mathrm{C}} / 3} c t}$ as $t \rightarrow \pm \infty$ ) and

$$
\begin{aligned}
Q=\frac{P_{2}(R)}{P_{1}(R)} & =c^{2} \frac{A_{\mathrm{C}} R^{6}-A_{\mathrm{P}} R^{3}+2 B}{3 H^{2} R^{6}} \\
& =1+c^{2} \frac{k R^{4}-A_{\mathrm{P}} R^{3}+B}{H^{2} R^{6}} \\
& \rightarrow 1 \quad\left\{\begin{array}{l}
\text { from above if } k>0 \\
\text { from below if } k \leq 0
\end{array}\right\} \text { as } R \rightarrow \infty .
\end{aligned}
$$

- For $k=-1$ or 0 , and for some cases of $k=1, H$ has a maximum value $H_{\max }$ at $R=R_{H_{\max }}$, where $d H / d R=$ $c^{2}\left(k R^{4}-A_{\mathrm{P}} R^{3}+B\right) / H R^{7}=0$. If $k=0, R_{H_{\max }}=\sqrt[3]{B / A_{\mathrm{P}}}$ and $H_{\max }=c \sqrt{A_{\mathrm{C}} / 3+A_{\mathrm{P}}^{2} / 3 B}$. As seen in Fig. 2 $H$ rises sharply from 0 at $R_{\min }$ to $H_{\max }$ at $R_{H_{\max }}$, then reverses and tails off asymptotically to $c \sqrt{A_{\mathrm{C}} / 3}$. One can show that $R_{\min } \sim \sqrt[3]{B / 2 A_{\mathrm{P}}}, R_{H_{\max }} \sim \sqrt[3]{B / A_{\mathrm{P}}}, R_{\mathrm{d}} \sim \sqrt[3]{2 B / A_{\mathrm{P}}}$, and $H_{\max } \sim c A_{\mathrm{P}} / \sqrt{3 B}$, as $B \rightarrow 0$ with $A_{\mathrm{C}}$ and $A_{\mathrm{P}}$ fixed. Thus, as $B \rightarrow 0$ with $A_{\mathrm{C}}$ and $A_{\mathrm{P}}$ fixed, $R_{\min }, R_{H_{\max }}$, and $R_{\mathrm{d}}$ are squeezed together closer and closer to 0 , and $H_{\max }$ grows without bound. This clearly is a recipe for an explosive postbounce inflation ending with a 'graceful exit' initiated by the onset of deceleration when $R$ reaches $R_{\mathrm{d}}$.

- As Fig. 2 exhibits, for $k=-1, k=0$, and $k=1$, graph a, the acceleration parameter $Q$, descending from $\infty$ at $R_{\min }$, passes through 1 at $R_{H_{\max }}$, bottoms out with a minimum value $Q_{\min }$ at $R_{Q_{\min }}$, where $d Q / d R=0$, then creeps slowly back to 1 as $R \rightarrow \infty$ (with a late pass through 1 if $k=1$ ). When $k=0, Q_{\min }=$ $\left.-\frac{1}{2}+\frac{3}{2} \sqrt{A_{\mathrm{C}} B /\left(A_{\mathrm{P}}^{2}+A_{\mathrm{C}} B\right.}\right)$ at $R_{Q_{\min }}=\left(B / A_{\mathrm{P}}+\sqrt{B^{2} / A_{\mathrm{P}}^{2}+B / A_{\mathrm{C}}}\right)^{\frac{1}{3}}$, which, with $A_{\mathrm{C}}$ and $A_{\mathrm{P}}$ fixed, are asymptotic respectively to $-\frac{1}{2}$ and $\sqrt[6]{B / A_{\mathrm{C}}}$ as $B \rightarrow 0$.

- If $k=1$ and $A_{\mathrm{C}}, A_{\mathrm{P}}$, and $B$ are such as to generate graph c of Fig. 1 , then for the section bounded by $R_{\min }$ and $R_{\max }$ the behavior of $R$ is cyclical, as Fig. 3 portrays graphically. 


\section{COSMOLOGICAL SOLUTIONS}

Study of solutions of the field equations (23) and (24) proceeds most smoothly when they are recast in terms of the variable $U:=S^{3}$, where $S:=R / R_{\min }$. The recast equations are together equivalent to

$$
\ddot{U}=3 c^{2} A_{\mathrm{C}} U-\frac{6 c^{2} k}{R_{\min }^{2}} U^{\frac{1}{3}}+\frac{3 c^{2} A_{\mathrm{P}}}{R_{\min }^{3}}
$$

and an equation for $\dot{U}^{2}$ that is essentially redundant. Initial conditions for $U$ are $U(0)=1$ and $\dot{U}(0)=0$, obtained from $S(0)=R(0) / R_{\min }=1$ and $\dot{S}(0)=\dot{R}(0) / R_{\min }=0$.

\section{A. Flat open universe $(k=0)$}

When $k=0$, so that space is perfectly flat, $R_{\text {min }}^{3}=-A_{\mathrm{P}} / A_{\mathrm{C}}+\sqrt{A_{\mathrm{P}}^{2}+A_{\mathrm{C}} B} / A_{\mathrm{C}}$ and it is straightforward to integrate Eq. (34), the result being, upon reversion from $U$ to $R$,

$$
R^{3}(t)=\left(R_{\min }^{3}+\frac{A_{\mathrm{P}}}{A_{\mathrm{C}}}\right) \cosh \left(\sqrt{3 A_{\mathrm{C}}} c t\right)-\frac{A_{\mathrm{P}}}{A_{\mathrm{C}}}
$$
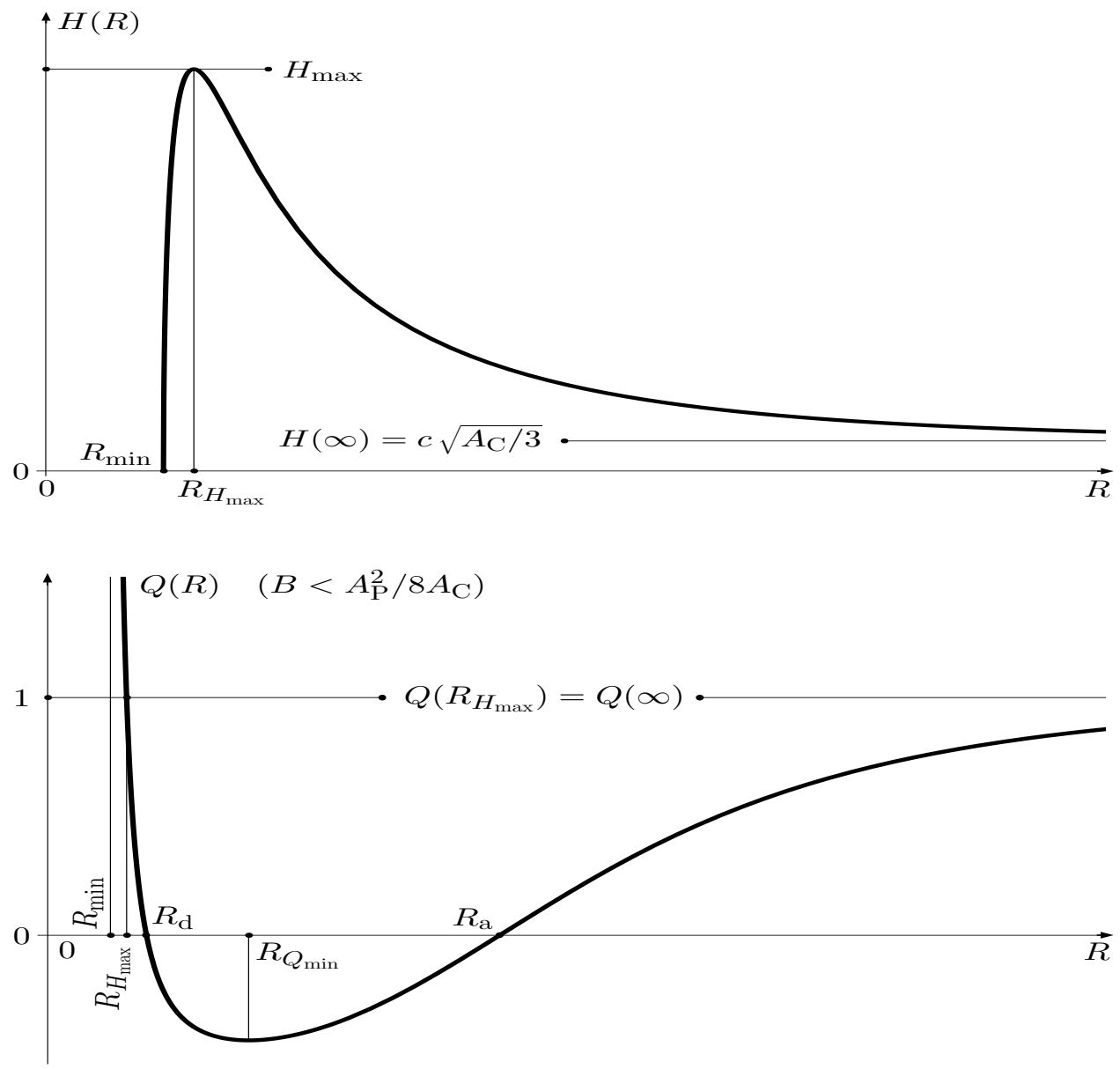

FIG. 2. Graphs of $H(R)$ and $Q(R)$ for $k=-1$ or 0 and generic positive values of the parameters $A_{\mathrm{C}}, A_{\mathrm{P}}$, and $B$, and for some cases of $k=1$, showing early stage inflation followed by a decline in $H$ to its asymptotic limit $H(\infty)=c \sqrt{A_{\mathrm{C}} / 3}$, and (for $B<A_{\mathrm{P}}^{2} / 8 A_{\mathrm{C}}$ ) transitions of $Q$ from inflationary acceleration to deceleration at $R_{\mathrm{d}}$ and back to acceleration at $R_{\mathrm{a}}$ to the asymptotic limit $Q(\infty)=1$. 
One can show that if $B \leq A_{\mathrm{P}}^{2} / 8 A_{\mathrm{C}}$, so that $R_{\mathrm{d}}$ and $R_{\mathrm{a}}$ exist, then $R_{\min }<R_{\mathrm{d}} \leq R_{\mathrm{a}}$. The postbounce times $t_{\mathrm{d}}$ and $t_{\mathrm{a}}$ at which $R=R_{\mathrm{d}}$ and $R=R_{\mathrm{a}}$ are the positive solutions of

$$
\cosh \left(\sqrt{3 A_{\mathrm{C}}} c t_{\{\mathrm{d}, \mathrm{a}\}}\right)=\frac{R_{\{\mathrm{d}, \mathrm{a}\}}^{3}+A_{\mathrm{P}} / A_{\mathrm{C}}}{R_{\min }^{3}+A_{\mathrm{P}} / A_{\mathrm{C}}} .
$$

The first graph in Fig. 4 displays the evolution of $R(t)$ for generic values of $A_{\mathrm{C}}, A_{\mathrm{P}}$, and $B$ with $B<A_{\mathrm{P}}^{2} / 8 A_{\mathrm{C}}$, showing the acceleration during the interval from $t=0$ to $t=t_{\mathrm{d}}$, the deceleration during the interval from $t_{\mathrm{d}}$ to $t_{\mathrm{a}}$, and the ultimately exponential acceleration after $t_{\mathrm{a}}$.

For times $t \approx 0$, Eq. (35) yields the approximation

$$
R^{3}(t) \approx R_{\min }^{3}\left(1+\frac{3 A_{\mathrm{C}}}{2} \frac{R_{\min }^{3}+A_{\mathrm{P}} / A_{\mathrm{C}}}{R_{\min }^{3}} c^{2} t^{2}\right),
$$

which produces the further approximation that

$$
R(t) \approx \hat{R}(t):=R_{\min }+\frac{A_{\mathrm{C}}}{2}\left(R_{\min }+\frac{A_{\mathrm{P}}}{A_{\mathrm{C}}} \frac{1}{R_{\text {min }}^{2}}\right) c^{2} t^{2},
$$

thus that $R(t)$ is approximately parabolic, opening upward from a vertex at $R_{\min }$, with an inflationary 'steepness' factor that grows as $c^{2} A_{\mathrm{P}} / 2 R_{\text {min }}^{2}$ as $R_{\text {min }} \rightarrow 0$.
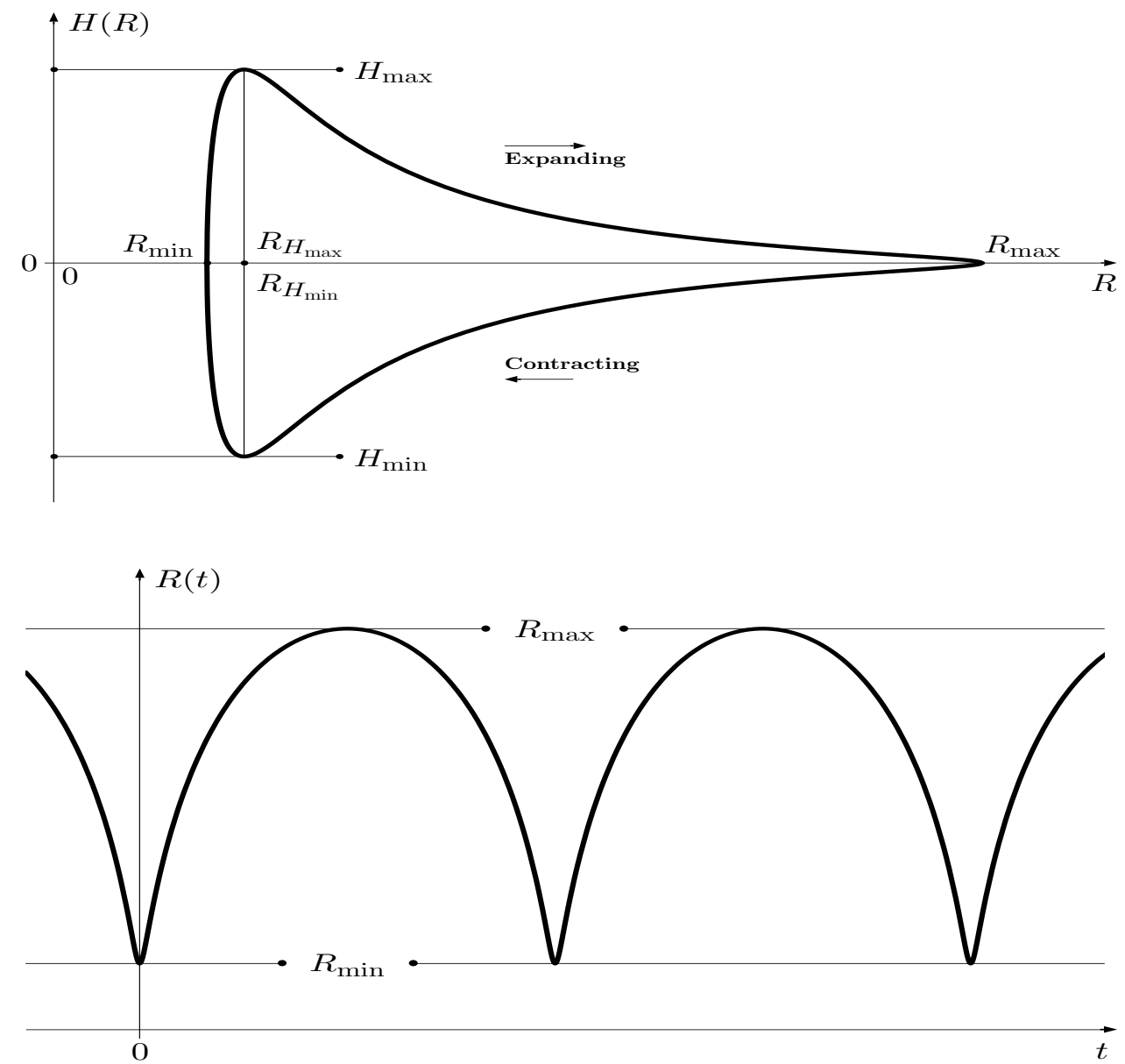

FIG. 3. Graphs of $H(R)$ and $R(t)$ for $k=1$ and generic positive values of the parameters $A_{\mathrm{C}}, A_{\mathrm{P}}$, and $B$ associated with graph $\mathrm{c}$ of Fig. 1 showing repetitive, identical periods of expansion and contraction, each beginning with a stage of rapid inflation from a bounce at $R=R_{\min }$, which is followed by a less rapid expansion to $R=R_{\max }$, then a mirror-image contraction to an ending stage of rapid deflation into the next bounce at $R=R_{\min }$. The graphed functions are related by $\dot{R}(t) / R(t)=: H(R(t))$. 

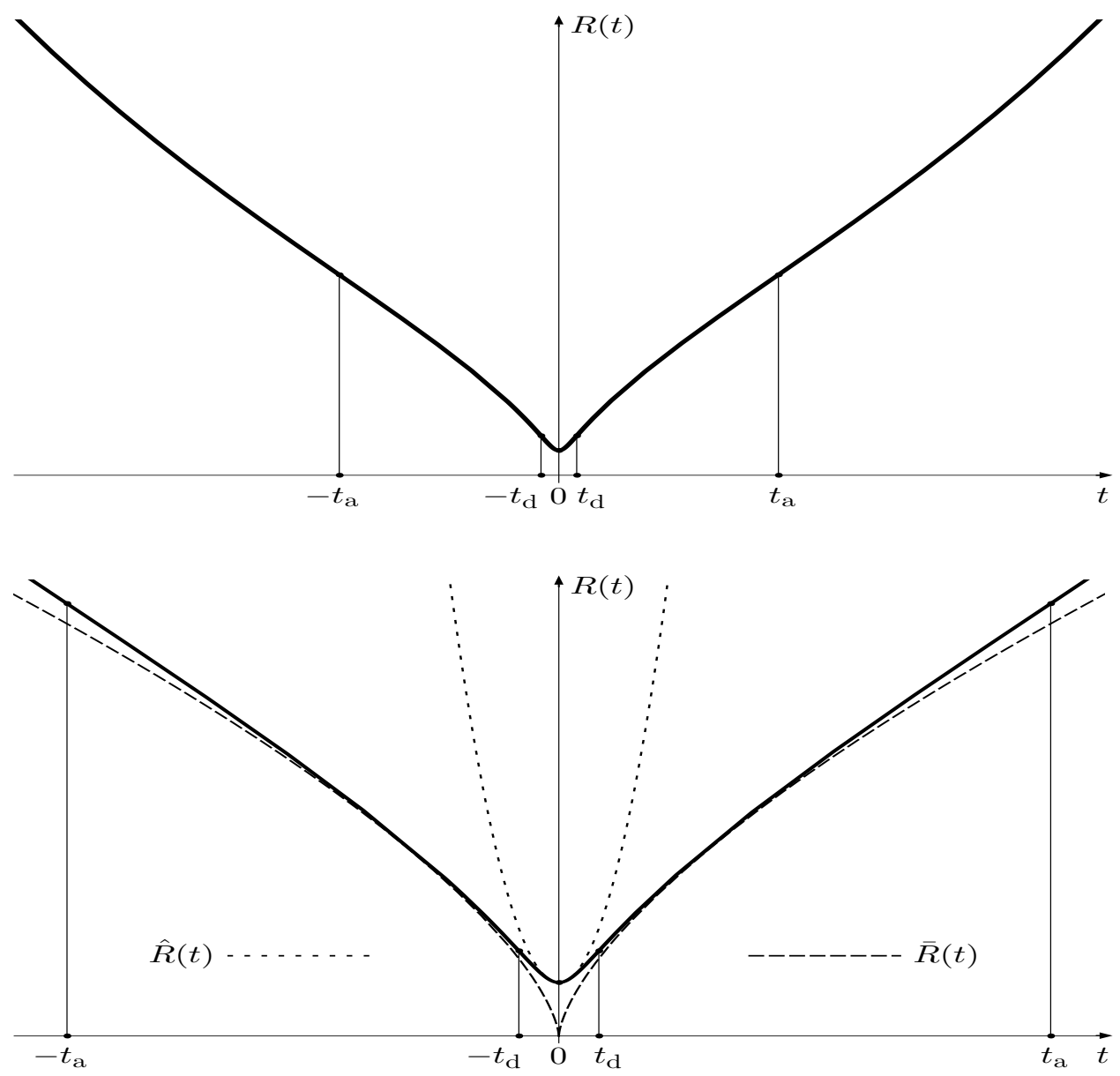

FIG. 4. Graph of $R(t)$ for $k=0$ and generic positive values of $A_{\mathrm{C}}, A_{\mathrm{P}}$, and $B$ with $B<A_{\mathrm{P}}^{2} / 8 A_{\mathrm{C}}$, showing early acceleration from $t=0$ to $t=t_{\mathrm{d}}$, deceleration from $t_{\mathrm{d}}$ to $t_{\mathrm{a}}$, and ultimately exponential acceleration after $t_{\mathrm{a}}$; truncated graph of $R(t)$ with approximations $\hat{R}(t)$ and $\bar{R}(t)$ as identified in Eqs. (38) and (40).

Equivalent to Eq. (37) is

$$
R^{3}(t)-R_{\min }^{3} \approx \frac{3 A_{\mathrm{C}}}{2}\left(R_{\min }^{3}+\frac{A_{\mathrm{P}}}{A_{\mathrm{C}}}\right)(c t)^{2}
$$

After $R(t)$ has inflated to the point that $R_{\min } / R(t) \ll 1,\left(R^{3}(t)-R_{\min }^{3}\right)^{\frac{1}{3}}$ will differ but little from $R(t)$, and then, for so long as $t$ remains small enough for the approximate Eq. (37) to hold, the approximation

$$
R(t) \approx \bar{R}(t):=\left[\frac{3 A_{\mathrm{C}}}{2}\left(R_{\min }^{3}+\frac{A_{\mathrm{P}}}{A_{\mathrm{C}}}\right)\right]^{\frac{1}{3}}(c t)^{2 / 3}
$$

will be valid. The second graph in Fig. 4 shows the approximations $\hat{R}(t)$ and $\bar{R}(t)$.

\section{B. Nonflat open $(k=-1)$ and closed $(k=1)$ universes}

When $k=-1$ or 1 , elementary symbolic solutions of Eq. (34) are unavailable, so one must resort to numerical integration. To enable the integration, numerical values must be chosen for the parameters other than $k$ that appear in Eq. (34), namely, $A_{\mathrm{C}}, A_{\mathrm{P}}$, and $B$ (implicitly through $R_{\min }$ ). An obvious way to effect this determination is to impose the requirement that the parameters provide a distance modulus curve that is a good fit to a Hubble diagram based on data from the observations of type Ia supernovae that revealed the acceleration of the expansion of the universe. Toward that end a number of definitions are in order: 
- $t_{0}$, the time of the present epoch, i. e., the time elapsed since the bounce at $t=0$.

- $H_{0}:=H\left(t_{0}\right)$, the present value of the Hubble parameter.

- $R_{0}:=c / H_{0}$, usually referred to as the 'Hubble radius' and as the 'radius of the visible universe'.

- $z:=R\left(t_{0}\right) / R-1$, the redshift function.

- $z_{\mathrm{d}}:=R\left(t_{0}\right) / R_{\mathrm{d}}-1$ and $z_{\mathrm{a}}:=R\left(t_{0}\right) / R_{\mathrm{a}}-1$, the redshifts associated with the transitions from acceleration to deceleration and back to acceleration.

- $\tilde{R}:=R / R\left(t_{0}\right)=1 /(1+z)$, a conventional notation.

- $\tilde{R}_{\min }:=R_{\min } / R\left(t_{0}\right)$ and $\tilde{R}_{\{\mathrm{d}, \mathrm{a}\}}:=R_{\{\mathrm{d}, \mathrm{a}\}} / R\left(t_{0}\right)$.

- $\lambda:=c / H_{0} R\left(t_{0}\right)=R_{0} / R\left(t_{0}\right)$, a useful parameter.

$$
\begin{aligned}
& \Omega_{\mathrm{C}}:=\frac{c^{2}}{3 H_{0}^{2}} A_{\mathrm{C}}, \\
& \Omega_{\mathrm{k}}:=-\frac{c^{2}}{H_{0}^{2} R^{2}\left(t_{0}\right)} k=-\lambda^{2} k, \\
& \Omega_{\mathrm{P}}:=\frac{2 c^{2}}{3 H_{0}^{2} R^{3}\left(t_{0}\right)} A_{\mathrm{P}}=\frac{2 H_{0} \lambda^{3}}{3 c} A_{\mathrm{P}},
\end{aligned}
$$

and

$$
\Omega_{\mathrm{B}}:=-\frac{c^{2}}{3 H_{0}^{2} R^{6}\left(t_{0}\right)} B=-\frac{H_{0}^{4} \lambda^{6}}{3 c^{4}} B
$$

With these definitions Eq. (23) is equivalent to

$$
H^{2}=H_{0}^{2}\left(\Omega_{\mathrm{C}}+\Omega_{\mathrm{k}} \frac{1}{\tilde{R}^{2}}+\Omega_{\mathrm{P}} \frac{1}{\tilde{R}^{3}}+\Omega_{\mathrm{B}} \frac{1}{\tilde{R}^{6}}\right)
$$

which upon evaluation at $t_{0}$ yields

$$
\Omega_{\mathrm{C}}+\Omega_{\mathrm{k}}+\Omega_{\mathrm{P}}+\Omega_{\mathrm{B}}=1 .
$$

On the other hand, Eq. (24) is equivalent to

$$
\begin{aligned}
\frac{\ddot{\tilde{R}}}{\tilde{R}} & =H_{0}^{2}\left(\Omega_{\mathrm{C}}-\frac{1}{2} \Omega_{\mathrm{P}} \frac{1}{\tilde{R}^{3}}-2 \Omega_{\mathrm{B}} \frac{1}{\tilde{R}^{6}}\right) \\
& =H_{0}^{2} \frac{2 \Omega_{\mathrm{C}} \tilde{R}^{6}-\Omega_{\mathrm{P}} \tilde{R}^{3}-4 \Omega_{\mathrm{B}}}{2 \tilde{R}^{6}},
\end{aligned}
$$

which yields, as roots of $\ddot{\tilde{R}}=0$,

$$
\tilde{R}_{\mathrm{d}}:=\left(\frac{\Omega_{\mathrm{P}}}{4 \Omega_{\mathrm{C}}}-\frac{\sqrt{\Omega_{\mathrm{P}}^{2}+32 \Omega_{\mathrm{C}} \Omega_{\mathrm{B}}}}{4 \Omega_{\mathrm{C}}}\right)^{\frac{1}{3}}
$$

and

$$
\tilde{R}_{\mathrm{a}}:=\left(\frac{\Omega_{\mathrm{P}}}{4 \Omega_{\mathrm{C}}}+\frac{\sqrt{\Omega_{\mathrm{P}}^{2}+32 \Omega_{\mathrm{C}} \Omega_{\mathrm{B}}}}{4 \Omega_{\mathrm{C}}}\right)^{\frac{1}{3}}
$$

(obtainable also from Eqs. (27) and (28) by substitution from Eqs. (41), (43), and (44)). 
To produce a curve to match against the data points of a Hubble diagram one needs the following standard formula for the luminosity distance $D_{\mathrm{L}}$ of a photon-emitting astronomical object at redshift $z$ :

Here

$$
D_{\mathrm{L}}(z)=(1+z) R\left(t_{0}\right) r_{\mathrm{k}}\left(\frac{c}{R\left(t_{0}\right)} \int_{0}^{z} \frac{1}{H(u)} d u\right) \text {. }
$$

$$
r_{\mathrm{k}}(\rho):= \begin{cases}\sinh \rho & \text { if } \quad k=-1 \\ \rho & \text { if } \quad k=0 \\ \sin \rho & \text { if } \quad k=1\end{cases}
$$

and, from Eq. (45) and the definition of $z$,

$$
\stackrel{*}{H}(z):=H_{0}\left[\Omega_{\mathrm{C}}+\Omega_{\mathrm{k}}(1+z)^{2}+\Omega_{\mathrm{P}}(1+z)^{3}+\Omega_{\mathrm{B}}(1+z)^{6}\right]^{\frac{1}{2}} .
$$

In terms of the parameter $\lambda$ the luminosity distance formula reads

$$
D_{\mathrm{L}}(z)=\frac{(1+z) c}{\lambda H_{0}} r_{\mathrm{k}}\left(\lambda \int_{0}^{z} \frac{d u}{\left[\Omega_{\mathrm{C}}+\Omega_{\mathrm{k}}(1+u)^{2}+\Omega_{\mathrm{P}}(1+u)^{3}+\Omega_{\mathrm{B}}(1+u)^{6}\right]^{\frac{1}{2}}}\right) .
$$

From $D_{L}$ one constructs the distance modulus $\boldsymbol{\mu}$, the difference between the apparent magnitude and the absolute magnitude of the object in question at redshift $z$; this reduces in the usual way to

$$
\boldsymbol{\mu}(z)=5 \log _{10}\left(\frac{D_{L}(z)}{10 \mathrm{pc}}\right)=25+\log _{10}\left(\frac{D_{L}(z)}{1 \mathrm{Mpc}}\right)
$$

From $\tilde{R}=1 /(1+z)$ follows $\dot{\tilde{R}}=-\dot{z} /(1+z)^{2}=-(d z / d t)(\tilde{R} /(1+z))$, thus $d t / d z=-1 /(1+z)(\dot{\tilde{R}} / \tilde{R})=-1 /(1+$ $z) \stackrel{*}{H}(z)$, which upon integration yields the following formula for the time $T(z)$ elapsed between an event occurring at redshift $z$ and the present time $t_{0}($ when $z=0)$ :

$$
\begin{aligned}
T(z) & =\int_{0}^{z} \frac{1}{(1+u)^{*}(u)} d u \\
& =\frac{1}{H_{0}} \int_{0}^{z} \frac{d u}{(1+u)\left[\Omega_{\mathrm{C}}+\Omega_{\mathrm{k}}(1+u)^{2}+\Omega_{\mathrm{P}}(1+u)^{3}+\Omega_{\mathrm{B}}(1+u)^{6}\right]^{\frac{1}{2}}} .
\end{aligned}
$$

The distance modulus $\boldsymbol{\mu}$ of Eq. (55) is the function to be fitted to the relative magnitudes data obtained from observations of type Ia supernovae. Using the SNe Ia data from the 182-member gold sample described in Sec. 3 of Ref. [15], the recent estimate $H_{0}=73.8 \mathrm{~km} \mathrm{~s}^{-1} \mathrm{Mpc}^{-1}$ obtained by Riess, et al. [16], and the Mathematica function NonLinearModelFit, I have carried out the fitting and reported the outcome in detail in Ref. [17]. The best fit, for which $\chi_{\text {red }}^{2}=0.869$, has $k=1, \Omega_{\mathrm{P}}=0.479, \Omega_{\mathrm{C}}=0.954, \Omega_{\mathrm{k}}=-0.433, \Omega_{\mathrm{B}}=-1.20 \times 10^{-196}, \lambda=0.658$, and $z_{\mathrm{a}}=0.585, z_{\mathrm{d}}$ being set somewhat arbitrarily at $1 \times 10^{65}$ to make the time at which the scale factor $R$ has doubled one hundred times from $R_{\text {min }}$ come out to approximately $10^{-35} \mathrm{~s}$. Figure 5 shows the Hubble diagram for this best fit, as well as for the corresponding fits for 76.2 and $71.4 \mathrm{~km} \mathrm{~s}^{-1} \mathrm{Mpc}^{-1}$, the upper and lower limits of the $3.3 \%$ confidence predicted in Ref. [16]. The evolution of the normalized scale factor $S=R / R_{\min }$ for the best fit is depicted graphically in Fig. 6.

Perhaps the most surprising prediction of this fitting to SNe Ia data is that when Eq. (57) is used to compute the time elapsed since the time of the 'big bounce' the result is 12.5 Gyr 2 This is at variance with the often quoted

\footnotetext{
${ }^{2}$ For $k=0$ or -1 the time elapsed is $12.7 \mathrm{Gyr}$, with $\chi_{\text {red }}^{2}=0.888$.
} 


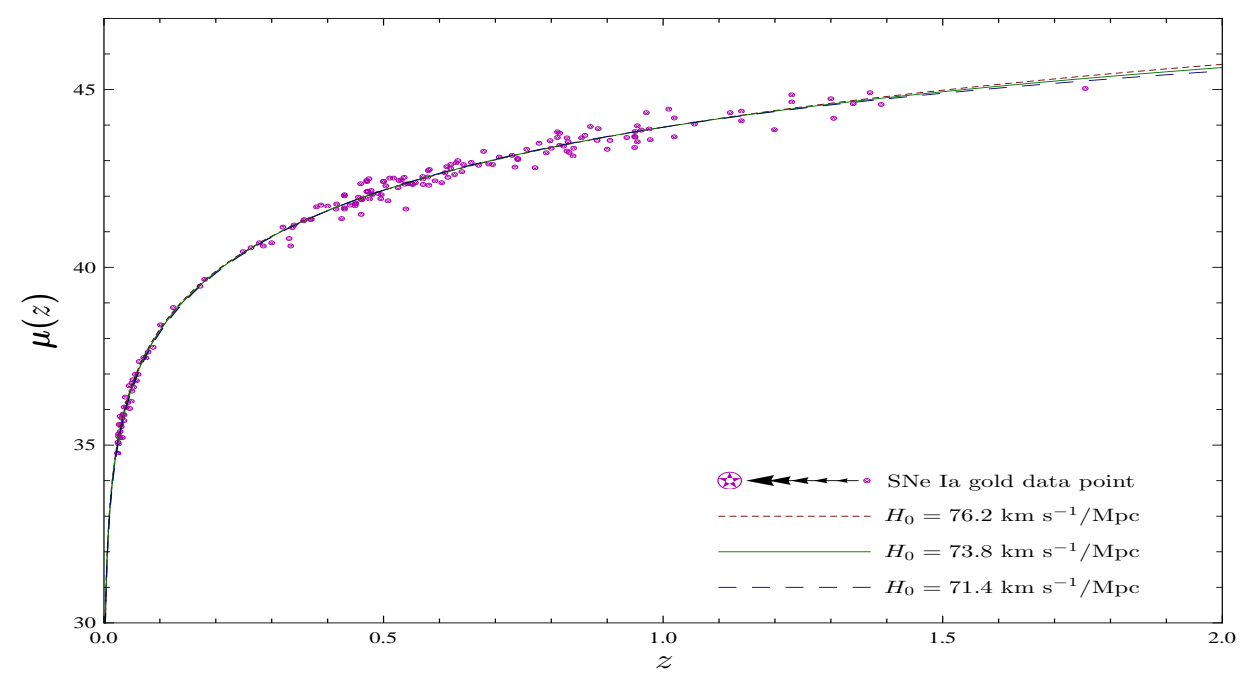

FIG. 5. Best-fit models of $\boldsymbol{\mu}(z)$ for $k=1$, plotted against the 182 SNe Ia gold sample data points they are fitted to. The curve for $H_{0}=73.8 \mathrm{~km} \mathrm{~s}^{-1} / \mathrm{Mpc}$ fits the 182 data points with $\chi_{\text {red }}^{2}=0.869$.

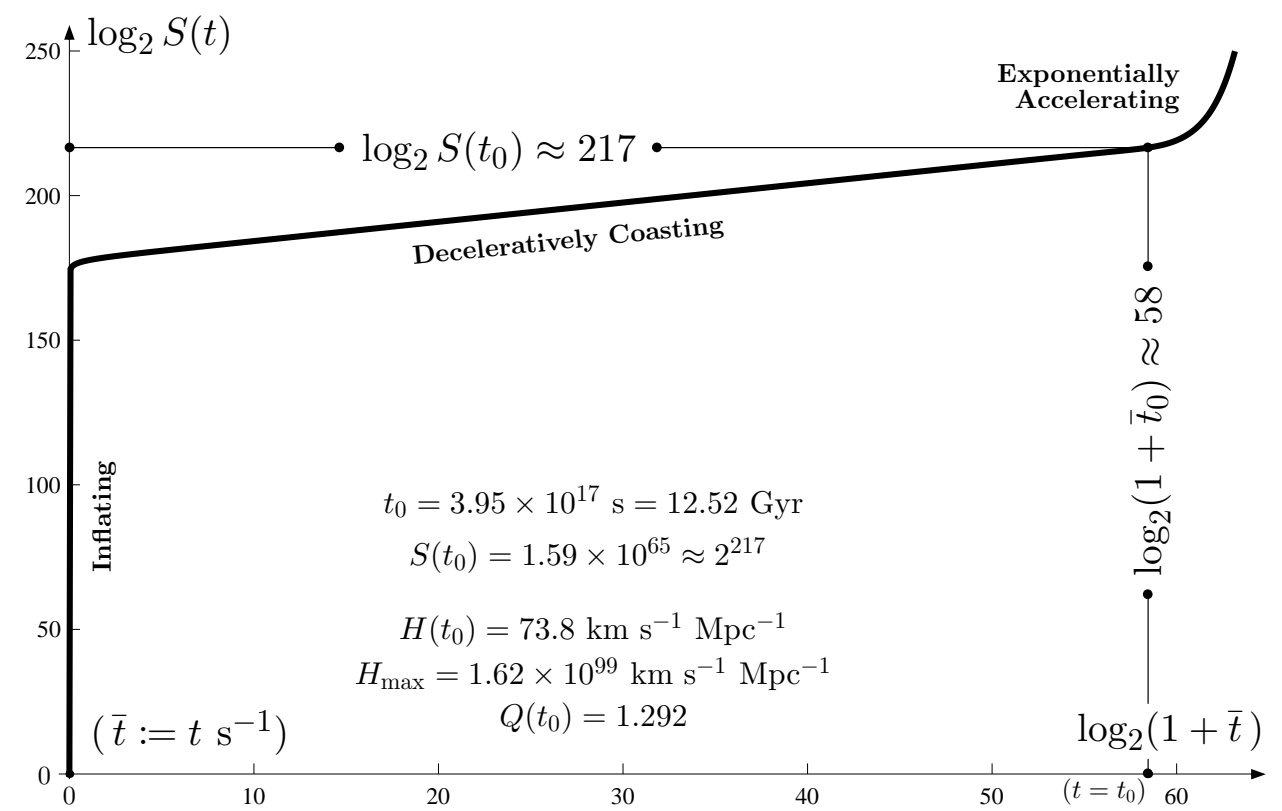

FIG. 6. Postbounce graph of $\log _{2} S(t)$ versus $\log _{2}(1+\bar{t})\left(\bar{t}:=t \mathrm{~s}^{-1}\right)$ for the best-fit solution with $H_{0}=73.8 \mathrm{~km} \mathrm{~s} \mathrm{Mpc}^{-1}$ and $k=1$. The early stage rapid inflation, after producing 178 doublings of the normalized scale factor $S\left(:=R / R_{\text {min }}\right)$ in the first second after the bounce, gives way smoothly to a period of uphill, decelerative 'coasting' (where the graph is nearly linear). Initially, the acceleration parameter $Q:=(\ddot{R} / R) /(\dot{R} / R)^{2}=(\ddot{S} / S) /(\dot{S} / S)^{2}$ is positive and huge $(Q(0)=\infty)$. In much of the coasting period $Q(t) \approx Q_{\min } \gtrsim-0.5$, which is reflected in the observation that during that time $\log _{2} S(t) \approx$ $178+\frac{217-178}{58-0} \log _{2}(1+\bar{t}) \approx 178+\frac{2}{3} \log _{2}(\bar{t})$, so that $S(t) \approx 2^{178} \bar{t}^{\frac{2}{3}}\left(S(t) \propto \bar{t}^{\frac{2}{3}} \Longrightarrow Q(t) \equiv-\frac{1}{2}\right)$. After the coasting era $Q(t)$ becomes positive again at redshift $z_{\mathrm{a}}=0.585$ (when $t=7.06$ Gyr and $\log _{2}(1+\bar{t})=57.6$ ), rising to 1.292 at $t=t_{0}$, and then to a maximum of 1.310 at $t=14.24$ Gyr before settling asymptotically to 1 as $t \rightarrow \infty$, the growth rate of $S(t)$ thus asymptotically becoming exponential as in a de Sitter universe. The corresponding graphs for the best-fit solutions with $H_{0}$ the same and $k=-1$ and $k=0$ are not appreciably different from this one.

interval of 13.7 Gyr for the time elapsed since the 'big bang'. I would point out, however, that when the corresponding fitting is carried out for the 'standard', or 'concordance', $\Lambda \mathrm{CDM}$ model of cosmology, with the Friedmann equation

$$
H^{2}=H_{0}^{2}\left(\Omega_{\Lambda}+\Omega_{\mathrm{k}} \frac{1}{\tilde{R}^{2}}+\Omega_{\mathrm{M}} \frac{1}{\tilde{R}^{3}}+\Omega_{\mathrm{R}} \frac{1}{\tilde{R}^{4}}\right)
$$


( $M$ for matter, $R$ for radiation) in place of Eq. (45), the best fit, with the same value of $\chi_{\text {red }}^{2}$, occurs when $k=1$, $\Omega_{\Lambda}=\Omega_{\mathrm{C}}, \Omega_{\mathrm{k}}=\Omega_{\mathrm{k}}, \Omega_{\mathrm{M}}=\Omega_{\mathrm{P}}$, and $\Omega_{\mathrm{R}}=6 \times 10^{-16}$, and also predicts 12.5 Gyr for the time elapsed since the big bang. One is left with the inference that the SNe Ia data, used alone, do not support, in either my model or the $\Lambda \mathrm{CDM}$ model, the older time of $13.7 \mathrm{Gyr}$ as the 'age of the universe', instead support an age younger by more than a billion years. This would seem to be a discrepancy that needs addressing 3

\section{DARK MATTER AND DARK 'ENERGY'}

The cosmological model derived in the preceding sections was based on the premise that the density of the active gravitational mass of all the matter in the universe is on balance negative. In the derivation no mechanism for production of this negative density was specified. In this section I show how 'drainholes' could provide such a mechanism, and in doing so would explain dark matter and explain away dark 'energy'.

\section{A. Drainholes}

The 'drainhole' model of a gravitating particle developed in Ref. [1] and proposed as a source for the negative, gravitationally repulsive mass density $\bar{\mu}$ in the action integral of Eq. (6) is a static, spherically symmetric, vacuum $(\mu=\bar{\mu}=0)$ solution of the same field Eqs. (17) that the cosmological model of the preceding sections is a solution of, but with the unorthodox choice $\phi \neq 0, \psi=0,5: 6$ This space-time manifold has come to be recognized as an early (apparently the earliest) example of what is now called by some a 'traversable wormhole' 19], and has been analyzed from various perspectives by others 20 34]. Its metric has the proper-time forms

$$
\begin{aligned}
c^{2} d \tau^{2} & =c^{2} d t^{2}-[d \rho-f(\rho) c d t]^{2}-r^{2}(\rho) d \Omega^{2} \\
& =\left[1-f^{2}(\rho)\right] c^{2} d T^{2}-\frac{1}{1-f^{2}(\rho)} d \rho^{2}-r^{2}(\rho) d \Omega^{2},
\end{aligned}
$$

where $T=t+\frac{1}{c} \int \frac{f(\rho)}{1-f^{2}(\rho)} d \rho$, and with $a:=\sqrt{n^{2}-m^{2}}$,

$$
f^{2}(\rho)=1-e^{-(2 m / n) \phi}
$$

and

$$
r(\rho)=\sqrt{(\rho-m)^{2}+a^{2}} e^{(m / n) \phi}=\sqrt{\frac{(\rho-m)^{2}+a^{2}}{1-f^{2}(\rho)}},
$$

in which

$$
\phi=\alpha(\rho)=\frac{n}{a}\left[\frac{\pi}{2}-\tan ^{-1}\left(\frac{\rho-m}{a}\right)\right]
$$

the parameters $m$ and $n$ satisfying $0 \leq m<n$. (The coordinate $\rho$ used here translates to $\rho+m$ in Ref. [1].) The shapes and linear asymptotes of $r$ and $f^{2}$ are shown in Fig. 7. Not obvious, but verifiable, is that $f^{2}(\rho) \sim 2 m / \rho$ as $\rho \rightarrow \infty$, which, together with $r(\rho) \sim \rho$ as $\rho \rightarrow \infty$, shows $m$ to correspond to the mass parameter of the Schwarzschild blackhole metric.

The shared metric of the cross sections of constant $t$ is $d \rho^{2}+r^{2}(\rho) d \Omega^{2}$. The 2-sphere at $\rho=2 m($ where $r(\rho)$ is a minimum) is the 'threshold' of the drainhole 'throat', crossed when transiting from the 'upper' region, where $\rho>2 m$, to the 'lower' region, where $\rho<2 m$, or vice versa. Its areal radius is $r(2 m)=n e^{(m / n) \alpha(2 m)}$, which increases

${ }^{3}$ An age of $13.2 \mathrm{Gyr}$ can be obtained, with not as good a fit, by taking $H_{0}=76.2 \mathrm{~km} \mathrm{~s}^{-1} \mathrm{Mpc}^{-1}$, the upper limit of the $3.3 \%$ confidence interval for $H_{0}$ predicted in Ref. [16], and $k=0$. At the lower limit $H_{0}=71.4 \mathrm{~km} \mathrm{~s}^{-1} \mathrm{Mpc}^{-1}$ and the oldest age obtainable is 12.3 Gyr, for $k=0$ or -1 .

${ }^{4} \boldsymbol{R}_{\alpha \beta}$ and $\boldsymbol{R}$ here are the negatives of those in Ref. [1].

5 The scalar field $\phi$ was presumed in Ref. [1] to satisfy the wave equation $\square \phi=0$ obtained from varying $\phi$ in the action integral. In retrospect that is seen to have been a redundancy, as the field Eqs. (9), which follow from variation of the metric alone, reduce when $\psi=0$ to $2(\square \phi) \phi . \alpha=0$, a consequence of which is $\square \phi=0$.

${ }^{6}$ Apparently the first to find static, spherically symmetric, vacuum solutions of Eqs. (7), albeit with the drainhole-excluding, orthodox choice $\phi=0, \psi \neq 0$ (descended directly from Einstein's assumption that inertial mass produces gravity), was I. Z. Fisher, in 1948. [18] 

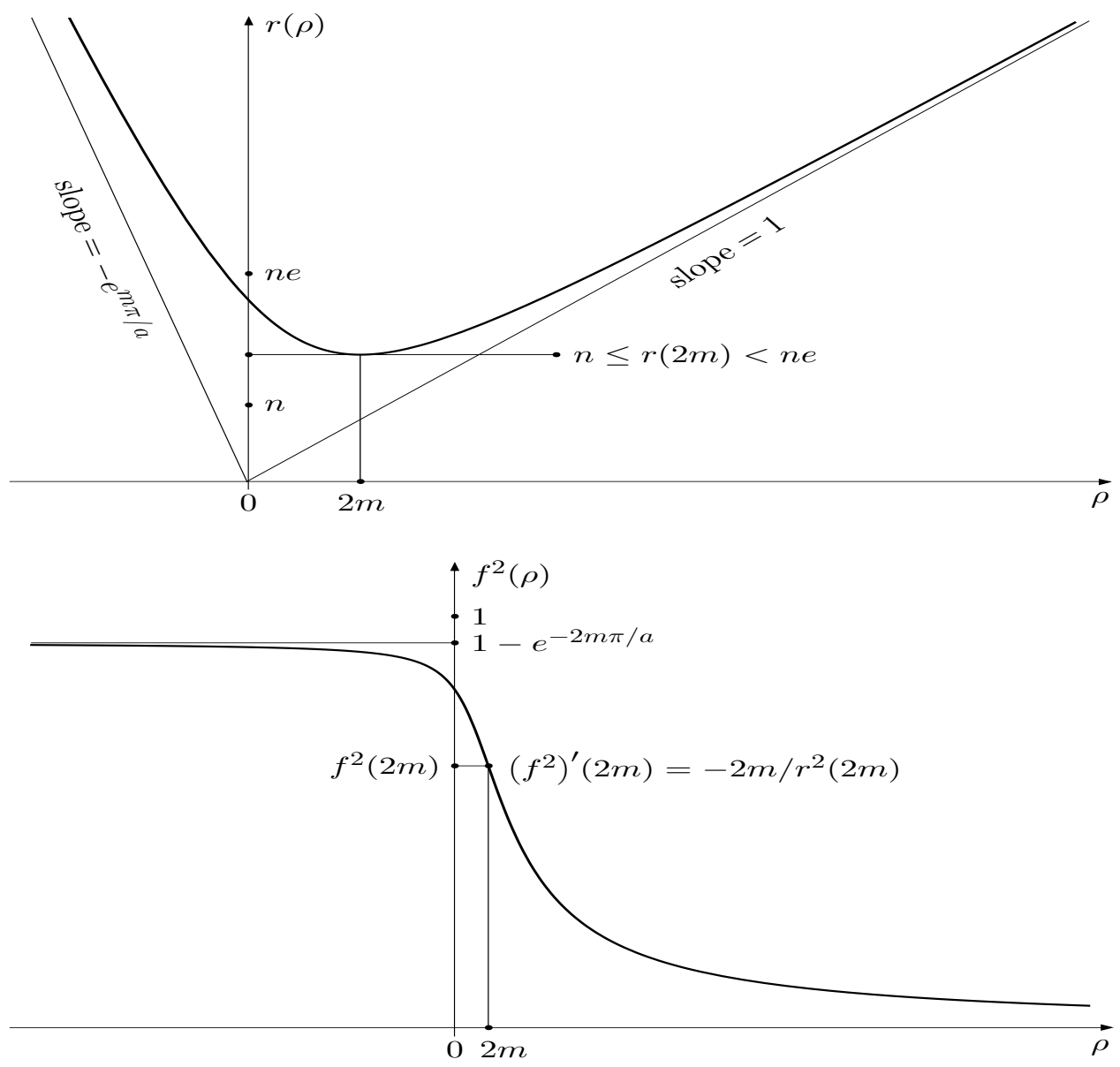

FIG. 7. Graphs of $r(\rho)$ and $f^{2}(\rho)$ for generic values of the parameters $m$ and $n\left(0 \leq m<n\right.$ and $\left.a:=\sqrt{n^{2}-m^{2}}\right)$.

monotonically from $n$ to $n e$ as $m$ increases from 0 to $n$. Thus the size of the threshold is determined primarily by $n$, and only ancillarily by $m$. Moreover, as indicated by the calculation that the contracted curvature tensor Ricci $=-2(d \phi \otimes d \phi)=-\left\{n^{2} /\left[(\rho-m)^{2}+a^{2}\right]^{2}\right\}(d \rho \otimes d \rho)$, the strength of $\phi$ 's 'contribution' to the space-time geometry is determined primarily by $n$ and is concentrated on the curvature of space, providing the negative spatial curvatures necessary for the open throat to exist. In accord with the restriction adopted here that the 'relaxant' scalar fields $\phi$ and $\psi$ in the action integral of Eq. (6) were not to be varied in deriving the field equations, one would not say that $\phi$ causes (i. e., is a source of) these spatial curvatures, but should instead say that $\phi$ allows such negative curvatures to exist and describes their configuration. This understanding helps disabuse one of the notion that geometrically and topologically unexceptionable space-time manifolds such as the drainhole are somehow a product of 'exotic' matter just because their Ricci tensors disrespect some 'energy condition' that traces back to Einstein's 1916 assumption that inertial-passive mass and active mass are the same.

Even when $m=0$, so that there is no gravity, the throat stays open, with $r(\rho)=\sqrt{\rho^{2}+n^{2}} 7$ It is not a great stretch to surmise that, whereas the parameter $m$ specifies the active gravitational mass of the (nonexotic) drainhole particle, the parameter $n$ specifies in some way its inertial rest mass. This speculation is supported by two considerations: first, as shown in Ref. [1], the total 'energy' of the scalar field $\phi$ lies in the interval from $n / 2$ to $n \pi / 2$, thus is essentially proportional to $n$; second, it would seem likely that the bigger the hole (thus the larger is $n$ ), the greater the force needed to make it move. A 'higgsian' way of expressing this idea is to say that the drainhole 'acquires' (inertial) mass from the scalar field $\phi$.

Because $r(\rho) \geq n>0$ and $f^{2}(\rho)<1$, the drainhole space-time manifold is geodesically complete and has no one-way event horizon, the throat being therefore traversable by test particles and light in both directions. The vector field $\partial_{t}+c f(\rho) \partial_{\rho}$ generates radial geodesics parametrized by proper time; with $f$ chosen as $-\sqrt{1-e^{-(2 m / n) \phi}}$ it is taken

\footnotetext{
7 The nongravitating drainhole is frequently referred to as the 'Ellis wormhole' [33, 34], as is occasionally the gravitating drainhole [26].
} 
to be the velocity field of a 'gravitational ether' flowing from $\rho=\infty$ on the high side (the upper region), down into the drainhole throat, across the threshold, out into the low side (the lower region), and onward to $\rho=-\infty$. The ether's radial acceleration is $\left(c^{2} f^{2} / 2\right)^{\prime}(\rho)$, which computes to $d^{2} \rho / d \tau^{2}=-c^{2} m / r^{2}(\rho)$ and therefore is strongest at the threshold, where $r(\rho)$ is at its minimum. Because the radial acceleration is everywhere aimed in the direction of decreasing $\rho$, the drainhole attracts test particles on the high side and repels them on the low side. Moreover, as demonstrated in Ref. 1] by means of an isometry of the manifold that exchanges the upper and lower regions with one another, whereas the upper region is asymptotic as $\rho \rightarrow \infty$ to a Schwarzschild manifold with (active gravitational) mass parameter $m$, the lower region is asymptotic as $\rho \rightarrow-\infty$ to a Schwarzschild manifold with mass parameter $\bar{m}=-m e^{m \pi / a}$, so the drainhole repels test particles more strongly on the low side than it attracts them on the high side, in the ratio $-\bar{m} / m=e^{m \pi / \sqrt{n^{2}-m^{2}}}$. It is this excess of negative, repulsive mass over positive, attractive mass that qualifies drainholes as candidates for explaining the accelerating expansion of the universe and doing away with the cosmological constant.

As mentioned in the Introduction, one can imagine that instead of a not well defined 'gravitational ether' it is space itself that flows into and through the drainhole 8 This substitution, which as noted in the Introduction is in accord with Einstein's insight that the concepts of space and of a gravitational ether are essentially interchangeable, should cause no alarm, for the very notion of an expanding universe already ascribes to space the requisite plasticity. There is, however, an ambiguity in that taking $f$ to be $\sqrt{1-e^{-(2 m / n) \phi}}$ instead of $-\sqrt{1-e^{-(2 m / n) \phi}}$ would have space (or the ether) appear to be flowing up through the drainhole rather than down. Insisting that the velocity of the flow be accelerative, not decelerative, would by fiat resolve the ambiguity in favor of the downward flow.

The discovery of the drainhole manifolds arose, in my case, from a search for a model for gravitating particles that, unlike a Schwarzschild space-time manifold, would have no singularity. Geodesic completeness and absence of event horizons resulted naturally from that requirement and the utilization of a minimally coupled scalar field to weaken the field equations. As shown in Ref. [1] a drainhole possesses all the geodesic properties a Schwarzschild blackhole possesses that do not involve its horizon or its singularity, having eliminated the horizon and replaced the singularity with a topological passageway to another region of space. Drainholes are able, therefore, to reproduce all the externally discernible aspects of physical blackholes (if such things exist) that Schwarzschild blackholes reproduce. That their low sides have never been knowingly observed (but in principle could be) is no more troubling than the impossibility of directly observing the insides of Schwarzschild blackhole horizons from external vantage points. For these reasons drainholes are more satisfactory than Schwarzschild blackholes as mathematical models of centers of gravitational attraction.

\section{B. Dark matter and dark 'energy' from drainholes}

A physical center of attractive gravity modeled by a drainhole could justifiably be called a 'darkhole', inasmuch as (as shown in Ref. [1]) it would capture photons (and other particles) that venture too close, but, unlike a blackhole, must with few exceptions eventually release them, either back to the attracting high side whence they came or down through the drainhole and out into the repelling low side. Thus one can imagine that at active galactic centers will be found not supermassive blackholes, but supermassive darkholes. At the center of our galaxy, for example, instead of a blackhole of Schwarzschild mass $m \approx 4 \times 10^{6} M_{\odot} \approx 5.91 \times 10^{6} \mathrm{~km}$ (in geometric units), and of horizon area $4 \pi(2 \mathrm{~m})^{2} \approx 1.75 \times 10^{15} \mathrm{~km}^{2}$, there might reside a drainhole the area $4 \pi r^{2}(2 \mathrm{~m})$ of whose threshold sphere would lie between $4 \pi n^{2}$ and $4 \pi(n e)^{2}$, with $n$ constrained only by having to exceed $m$. As measured by the bounds $n$ and $n e$ on the areal radius $r(2 m)$, and the corresponding bounds $c^{2} m / n^{2}=c^{2}(m / n)(1 / n)$ and $c^{2} m /(n e)^{2}=c^{2}(m / n)\left(1 / e^{2} n\right)$ on the maximum radial acceleration $c^{2} m / r^{2}(2 m)$, with $m$ constrained by $0 \leq m<n$, such an object could be of any size and could be weakly gravitating for its size $(m \ll n)$, strongly gravitating for its size $(m \approx n)$, or anything in between 9

There is, however, more to be said. A central tenet of the general theory of relativity is that every object that gravitates, no matter how large or how small, manifests itself in (more fundamentally, is a manifestation of) a departure of the geometry of space-time from flatness. If such an object has other, nongravitational properties, these must be either incorporated in or additional to the underlying geometric structure. Believing that the drainhole model provides the best presently available description of a gravitating particle's geometry, I adopt the hypothesis that every such elementary gravitating object is at its core an actual physical drainhole - these objects to include not only elementary constituents of visible matter such as protons and neutrons (or perhaps, more elementarily, quarks), but also the unseen particles of 'dark matter' whose existence is at present only inferential. Moreover, I assume that

\footnotetext{
8 This idea has been applied in Ref. 35] to the interpretation and visualization of blackholes, with interesting results.

${ }^{9}$ A recent paper explores the possibility of observationally distinguishing between a Schwarzschild blackhole and a nongravitating 'Ellis wormhole' at a galactic center. [36]
} 
visible matter and the primordial matter of my cosmological model are one and the same, and that drainholes with no additional properties constitute the continuously created matter of the model.

The pure, isolated drainhole described by Eqs. (59 63) is an 'Einstein-Rosen bridge' connecting two otherwise disjoint 'subuniverses' [37], each of which, if evolving, would by itself consume for its description all the resources of a Robertson-Walker metric. Nonisolated drainholes presumably could exist not only as 'bridges' between our subuniverse and another (or multiple others), but also as 'tunnels' from one place in our subuniverse to another, possibly quite distant from the first by every route that doesn't pass through a tunnel. Both types could contribute to the positive and the negative mass densities $\mu$ and $\bar{\mu}$ in our subuniverse, each bridge drainhole contributing to $\mu$ or to $\bar{\mu}$, but not to both, each tunnel drainhole contributing both to $\mu$ by way of its gravitationally attractive entrance portal and to $\bar{\mu}$ by way of its gravitationally repulsive exit portal. Tunnel drainholes are easy enough to visualize in abundance as topological holes into which flowing space disappears, only to reappear elsewhere in our subuniverse, in analogy with rivers that go underground and surface somewhere downstream. Having both portals located in our subuniverse, tunnel drainholes would have properties we could take fully into account. Bridge drainholes, on the other hand, with only one side in our subuniverse would have properties dependent in part on circumstances in other subuniverses, circumstances beyond our ken. I assume, therefore, that tunnel drainholes contribute to $\mu$ and to $\bar{\mu}$, and bridge drainholes contribute to neither, which leaves our subuniverse gravitationally self-contained, but possibly connected to other subuniverses of a 'multiverse' by way of flowless bridge drainholes (nongravitating 'Ellis wormholes').

Lacking for the present a full mathematical description of these tunnel drainholes, one can nevertheless proceed under the assumption that they exist and are characterized by parameters $m$ and $n$ related as in an isolated bridge drainhole. Every particle of gravitating matter, whether in the 'primordial' (P-matter) category or the 'continuously created' (C-matter) category, is then at its core one of these tunnels, and it becomes a question of relating $m$ and $n$ to the present-epoch densities $\mu_{\mathrm{P}, 0}, \bar{\mu}_{\mathrm{P}, 0}, \mu_{\mathrm{C}, 0}$, and $\bar{\mu}_{\mathrm{C}, 0}$. A way to do this is developed in Ref. [17] (Sec. VI.B), and will not be repeated here.

\section{ISSUES AND OBSERVATIONS}

The conceptual basis of the model of the cosmos developed in the preceding sections differs in many respects from that of the standard, concordance model. These differences and their consequences, each stemming directly or indirectly from disallowing Einstein's implicit assumption that inertial mass produces gravity, bring up a number of issues, some of which have been discussed above to greater or lesser extent, others of which have been passed over. In this section I identify and comment on several of them. For ease of reference I will call my model the PCDM model (P for primordial, $\mathrm{C}$ for continuously created, $\mathrm{D}$ for drainhole, $\mathrm{M}$ for matter) and the concordance model the $\Lambda \mathrm{CDM}$ model ( $\Lambda$ for $\Lambda, \mathrm{C}$ for cold, $\mathrm{D}$ for dark, $\mathrm{M}$ for matter).

\section{A. Gravity and passive-inertial mass}

As noted at the beginning, Newton's law of action and reaction allows the inference that the ratio of active gravitational mass to passive-inertial mass is the same for all 'bodies', that is, for all matter in bulk. Because Newton's theory describes gravity as well as it does, this conclusion must be at least approximately correct. Even if exact, however, it is only a statement about a numerical ratio of quantities that conceptually have nothing to do with one another: the generation of a gravitational field on the one hand, the resistance to being accelerated by a field of any sort on the other. I pointed out in Sec. III that Einstein's theory of gravity in the vacuum has in it no concept of inertial mass. Subsequently I suggested (in Sec. VIA) that the concept might now have been brought into the vacuum theory as the parameter $n$ of the drainhole model. If so, then the particulars of the drainhole model make it quite clear that inertial mass and active gravitational mass are entirely independent concepts. Specifically, as shown in [1], when the active mass parameter $m$ is 0 but $n \neq 0$, test particles follow the geodesics of the spatial metric $d \rho^{2}+\left(\rho^{2}+n^{2}\right) d \Omega^{2}$. These geodesics bend around the drainhole, so give the appearance that the test particles are acted on gravitationally by the drainhole. But a test particle follows such a geodesic at constant velocity, which can in fact be 0, leaving the test particle sitting at rest for all time wherever it happens to be - clearly not under any gravitational influence. In this way the drainhole model delineates the distinction between the curvature of space, on one hand, and gravity (the 'curvature of time') on the other.

If passive-inertial mass is not a source of gravity, then as said in Sec. III Einstein's 'energy-tensor of matter' $T^{\alpha \beta}:=\left(\rho+p / c^{2}\right) u^{\alpha} u^{\beta}-p g^{\alpha \beta}$ has no role to play in the field equations of gravity, so one loses the alluring implication that $0=T_{\alpha}{ }^{\beta}: \beta=\left(\left(\rho+p / c^{2}\right) u^{\alpha} u^{\beta}\right)_{: \beta}-p_{. \beta} g^{\alpha \beta}$, which implication Einstein interpreted as saying that a consequence of his field equations was that "the equations of conservation of momentum and energy... hold good for the components 
of the total energy", and cited as "the strongest reason for the choice" of his equations ( $\$ 16$ of Ref. [5]). The loss of this implication does not, of course, keep us from asserting that $T_{\alpha}{ }^{\beta}: \beta=0$; it only requires that we look elsewhere for a justification. But one must recognize that for (Einstein's) $T_{\alpha}^{\beta}: \beta=0$, however arrived at, to be interpreted as a proposition about conservation of momentum and energy $\rho$ must be the density of inertial mass, not, as Einstein assumed, the density of active gravitational mass.

All too frequently there appear in the popular press accounts of astronomers 'weighing' the universe or 'weighing' a galaxy. One can object, of course, to the presumably intentional blurring of the distinction between inertial mass and weight, but a larger objection is that such accounts leave the reader with the false impression that determining an entity's active gravitational mass is known to be equivalent to determining its passive-inertial mass (and therefore its weight). Only if one can prove the existence of a universal proportionality between the two can one legitimately claim to have 'weighed' the universe or a galaxy, and even then only indirectly. Unless and until there is such a proof, pretending that the universe or a galaxy has been weighed spreads ignorance, not knowledge. The most elementary unit of knowledge is a distinction made - a fact every digital computer is based on. Conversely, the most elementary unit of ignorance is a distinction not made.

\section{B. Choice of a variational principle for gravity}

The step up in Sec. III from the nonrelativistic variational principle $\delta \int\left(|\nabla \phi|^{2}+8 \pi \kappa \mu \phi\right) d^{3} x=0$ that generates the Poisson equation $\nabla^{2} \phi=4 \pi \kappa \mu$ to the relativistic principle $\delta \int\left(\boldsymbol{R}-\frac{8 \pi \kappa}{c^{2}} \mu\right)|g|^{\frac{1}{2}} d^{4} x=0$ that generates the equations $\boldsymbol{R}_{\alpha \beta}-\frac{1}{2} \boldsymbol{R} g_{\alpha \beta}=-\frac{4 \pi \kappa}{c^{2}} \mu g_{\alpha \beta}$ is an application of Occam's razor, and as such can only be justified retrospectively, by its consequences. Alone, this principle has, through its Euler-Lagrange equations, little to say about cosmology, but when modified by inclusion of the cosmological constant $\Lambda$ it says something significant, namely, that there is gravitationally repulsive matter in the universe (disguised as $-\Lambda$ ), and there can be more of it than there is of gravitationally attractive matter. This is a consequence that has consequences, but to get them all requires the next step up, to the action integrand of Eq. (6) which has in it in addition to the positive and negative mass densities $\mu$ and $\bar{\mu}$ the (gradients of the) scalar fields $\phi$ and $\psi$, which are considered as auxiliary 'relaxants' not to be varied. What justifies their inclusion?

A defect of the Einstein vacuum field equations, generally unrecognized as such, is that they produce as their basic model for a gravitating particle a manifold that exhibits no spatial curvature when viewed from the perspective of observers free-falling from rest at infinity. (This is seen in Eq. (59), in which $t$ coincides with the proper time of such observers and on $t=$ constant cross sections of the Schwarzschild manifold the metric is that of (flat) euclidean 3-space, viz., $d \rho^{2}+\rho^{2} d \Omega^{2}$.) Inclusion of the scalar field $\phi$ in the action integral of Eq. (6) corrects this defect, as it allows the constant- $t$ cross sections of the drainhole model to have the negative spatial curvatures characteristic of the drainhole, and therefore admits the possibility that the space we reside in is something other than euclidean 3-space.

The inclusion of a second scalar field $\psi$, coupled to the metric with polarity opposite to that of $\phi$ 's coupling, is dictated in the first instance by the absence, when Einstein's assumption of equivalence between passive-inertial mass and active gravitational mass is denied, of any real reason to choose one polarity over the other. In the second instance it is dictated by the observation that, although the presence of $\phi$ but not of $\psi$ was required for the derivation of the drainhole model, in the construction of the cosmological model absence of $\psi$ would entail absence from Eq. (22) of the $\dot{\beta}^{2}$ term, in which case Eq. (22) would turn from true to false once $R(t)$ surpassed $\sqrt[3]{B / A_{\mathrm{P}}}$ (which is $R_{H_{\max }}$ when $k=0$ ). Moreover, if the shoe were on the other foot and the $\dot{\alpha}^{2}$ term were absent, then Eq. (22) would be false when $R(t)$ was less than $\sqrt[3]{B / A_{\mathrm{P}}}$. Thus both $\phi$ and $\psi$ are needed, coupled to the geometry with opposite polarities. One notices that $\alpha$ and $\beta$, appearing only in the combination $\dot{\alpha}^{2}-\dot{\beta}^{2}$, can be individuated only by an arbitrary allocation of the righthand side of Eq. (22) that preserves positives and negatives, such as $\dot{\alpha}^{2}=c^{2} B / R^{6}+h$ and $\dot{\beta}^{2}=c^{2} A_{\mathrm{P}} R^{3} / R^{6}+h$, where $h$ is a nonnegative function of $t$.

That the combination $2 \phi^{\cdot \gamma} \phi_{\cdot \gamma}-2 \psi^{\cdot \gamma} \psi_{\cdot \gamma}$ in the action integrand of Eq. (6) is the real part of $2 \chi^{\cdot \gamma} \chi_{. \gamma}$, where $\chi:=\phi+i \psi$, suggests that space-time as seen here might be a restriction of a more general, at least partly complexified space-time geometry.

\section{Inflation and the 'big bounce'}

Further evidence that generation of a realistic cosmological model depends on inclusion of the scalar field $\phi$ with the so-called 'ghost' or 'phantom' coupling to geometry comes from the recognition that without it there is a 'bang' singularity at $R_{\min }=0$ and no inflation to follow, whereas with it there is a nonsingular bounce at $R_{\text {min }}>0$, followed 
by inflation. As is evident from the definition of $P_{1}(R)$ in Eq. (25), as well as from examining Fig. 1 for $R_{\text {min }}$ to be positive it is necessary and sufficient that the integration constant $B$ be positive (otherwise $P_{1}(0)=-B \geq 0$, which would make $R_{\min }=0$ ). Also evident is that the smaller a positive $B$ is, the closer $R_{\min }$ is to 0 ; in fact, as noted in Sec. IV $R_{\text {min }} \sim \sqrt[3]{B / 2 A_{\mathrm{P}}}$ as $B \rightarrow 0$. Moreover, it is clear that the term $2 B / 3 R^{6}$ in Eq. (24) is the term that produces rapid inflation when $R$ is small by making $\ddot{R}$ large, but only when $B>0$. And because $B-A_{\mathrm{P}} R_{\min }^{3} \sim B-A_{\mathrm{P}}\left(B / 2 A_{\mathrm{P}}\right)=B / 2$ as $B \rightarrow 0$, positivity of $B$ demands that the $\dot{\alpha}^{2}$ term be present in Eq. (22), thus that the scalar field $\phi$ be present in the field equations, coupled to the space-time geometry with the unconventional polarity. Let us note, moreover, that here there is no mention of a 'slow roll down a potential', a common element in many proposed inflationary scenarios involving an 'inflaton' scalar field. Indeed, that $\phi$ is treated merely as an aid in describing the space-time geometry rather than as a 'physical source' of the geometry makes the notion of introducing a potential function for $\phi$ irrelevant.

\section{Dark matter, dark 'energy', and the 'Cosmological Constant Problem'}

In the $\Lambda$ CDM model dark matter is lumped with baryonic matter in $\Omega_{\mathrm{M}}$ of the Friedmann equation (Eq. (58)) as primordial matter whose density is proportional to $1 / R^{3}$. The unseen, unknown stuff in $\Omega_{\Lambda}$, whose density has no $R$ dependence, is called dark 'energy' in compliance with Einstein's assumption that inertial mass, therefore energy, is a source of gravity. In the PCDM model only baryonic matter is treated as belonging to the primordial $\Omega_{\mathrm{P}}$ sector with $1 / R^{3}$ density dependence, dark matter being located in the $\Omega_{\mathrm{C}}$ sector with density kept constant by continuous creation of the dark C-matter. And there, instead of dark 'energy' represented by a cosmological constant $\Lambda$ required by the data to be many orders of magnitude too small to be consistent with the 'vacuum energy' proposed as its source, one finds the gravitationally attractive dark matter particles accompanied by their gravitationally repulsive back sides, their net effect represented by the constant $A_{\mathrm{C}}$ of Eq. (21), functionally equivalent to $\Lambda$ and with the requisite smallness readily producible by drainholes 10 This interpretation of 'dark energy' as the back side of dark matter provides a self-consistent solution of the so-called 'Cosmological Constant Problem'.

\section{E. Ratio of dark matter to baryonic matter}

Because in the $\Lambda$ CDM model the densities of dark matter and baryonic matter are both proportional to $1 / R^{3}$, the ratio of their densities is constant in time. Estimates of that ratio obtained from observations of visible structures such as galaxies and galactic clusters and superclusters as they exist at the present epoch in the vicinity of our galaxy are therefore used in studies of the formation of these structures in the distant past. In the PCDM model, on the other hand, the dark matter density is held constant through continuous creation of C-particles, so the ratio $\mu_{\mathrm{C}} / \mu_{\mathrm{P}}$ of dark C-matter density to baryonic P-matter density grows in proportion to $R^{3}$. Consequently, the value of this ratio at the epoch corresponding to redshift $z$ is $\gamma(z)=\gamma_{0} /(1+z)^{3}$, where $\gamma_{0}$ is the ratio at present. Taking this dependence of the ratio on redshift as real would no doubt influence the outcomes of studies of structure formation.

\section{F. Continuous creation of C-matter tunnels}

The stipulation that the net density $\mu_{\mathrm{C}}+\bar{\mu}_{\mathrm{C}}$ of C-matter stay fixed while the universe is expanding requires that the drainhole tunnels considered to be the particles of C-matter come continuously into existence. By what mechanism might this happen? Wheeler's notion of a 'quantum foam' of wormholes popping into and out of existence would not provide a satisfactory explanation, for in order to keep the density constant each newly created tunnel must either remain in existence or else upon dying be multiply replaced by new ones. A more useful idea is that the tunnels arise from the stretching of space as the universe expands. Once started, this would be a self-sustaining process, each new tunnel causing by its excess of repulsion over attraction additional stretching that would generate additional tunnels. Such a process, moreover, could be expected to produce tunnels in a given region of space at a rate proportional to the rate of increase of the volume of that region, thus maintaining a constant tunnel particle density. Not only that, these tunnels, once in existence, might very well themselves expand along with the stretching of space, which they could do without altering their active gravitational masses, thus without changing the net density. As to how the process could begin, one looks to the primordial P-matter tunnels, presumed to have always been present, pushing the

10 For the best fit value 0.954 of $\Omega_{\mathrm{C}}$ mentioned in Sec. $\mathrm{VB} A_{\mathrm{C}}=\left(3 H_{0}^{2} / c^{2}\right) \Omega_{\mathrm{C}}=1.821 \times 10^{-52} \mathrm{~m}^{-2}$. Sec. VI.B of Ref. [17] shows how drainholes can produce such a value. 
universe toward expansion. In the postbounce era the C-particles would add their contribution, the ultimate result being an acceleration of the expansion. In the prebounce era the C-particles, abundant in the distant past, would be dying out with the shrinking of space as the universe contracted into the bounce 11

If the primordial P-matter has 'always' existed, then the question of what 'caused' it to exist is better left to the philosophers. Conceivably, however, the P-matter tunnels came into existence as dark matter particles the same way the C-matter tunnels did, but in the inflationary space-stretching era after the bounce. Only later would their entrance portals have undergone modifications that turned them into particles of visible matter. The really fundamental unanswered question, though, is how did space and time come to exist. In fact, the question can be reduced to the existence of space alone, for the Kaluza-Weyl theory described in Ref. 38] (and more succinctly in Ref. [39]) is based on a construction that produces time (thus space-time) from three-dimensional space and, repeated, produces a secondary time (thus space-time-time) from space-time. And at the space-time stage of that theory there occurs naturally a model of an expanding drainhole in an expanding universe which repels on the low side more strongly than it attracts on the high side and needs no auxiliary scalar field for its description [42].

It cannot be ruled out that some C-particles (and some P-particles as well) have active gravitational mass $m=0$, but, because $n \neq 0$, have nonzero inertial mass. Being without electric charge, and possessing some rotational properties as suggested in Ref. 31] to be possible, such tunnel particles might be produced in abundance and could serve as models for the ubiquitous cosmic neutrinos. If such models are realistic, then analyses that use cosmological observations to put upper bounds on the neutrino mass scale (the analysis in Ref. [43] using observations of galaxy clustering, for example) say nothing about the inertial masses of neutrinos, instead 'constrain' only their active gravitational masses, already assumed in the models to be zero. Even if these models are not realistic, in the absence of any demonstrable relation for neutrinos between the two kinds of mass such analyses based on gravitational effects cannot be presumed to constrain their inertial masses.

One other possibility to consider is that as time goes on a fraction of the C-particles acquire the trappings of ordinary matter and are then able to join in the formation of visible-matter structures. This would offer a way for the universe to avoid a cold, dark ending to its exponentially accelerating expansion.

\section{G. Voids, walls, filaments, and nodes}

The PCDM model, just as the $\Lambda \mathrm{CDM}$ model, treats matter as uniformly distributed in space, an apparently reasonable assumption on a large enough scale. Observationally, however, a major fraction of space appears to comprise voids nearly empty of visible matter, separated by walls, filaments, and nodes in which resides most of the matter, both the dark and the visible. The formation of this cellular structure is generally considered to be an evolutionary product of early small fluctuations in the density of primordial matter. Explanations along that line are attempts to realize what Peebles has called his "perhaps desperate idea ... that the voids have been emptied by the growth of holes in the mass distribution" [44]. If besides matter that attracts gravitationally there is also matter that repels, then that idea can be reinterpreted and the desperation perhaps alleviated, by the simple observation that, whereas attractive matter wants to congregate, repulsive matter is reclusive, pushing all matter, attractive or repulsive, away from itself.

In the PCDM model each tunnel particle would presumably be created with its entrance and its exit close to one another in the ambient space. In the ambient space the entrance would attract the exit, but the exit would repel the entrance more strongly, so the two portals would drift apart. Apply this to a multitude of such particles and you might expect to see the exits spread themselves over regions from which they had expelled the entrances, regions therefore devoid of attractive matter. The entrances, on the other hand, being brought together by both their mutual attractions and the repulsion from the exits, would aggregate into walls, filaments, and nodes on the boundaries between the void regions, just as is seen in the real universe. What is more, the walls, filaments, and nodes so created would likely be, in agreement with observation, more compacted than they would have been if formed by gravitational attraction alone, for the repulsive matter in the voids would increase the compaction by pushing in on the clumps of attractive matter from many directions with a nonkinetic, positive pressure produced by repulsive gravity, a pressure not to be confused with the negative pseudo-pressure conjectured in the confines of Einstein's assumption to be a producer of repulsive gravity. In this way the PCDM model can explain qualitatively the observed cellular structure of the universe with minimal reliance on preexisting fluctuations in the primordial matter density — it is conceivable that creation of tunnel entrance-exit pairs at random locations in random orientations during the inflationary phase

11 The once seemingly moribund idea of a universe held in a 'steady state' by continuous creation of matter through the agency of a 'negative-energy' scalar field [40, 41] has been to a considerable extent reinvigorated by the recognition that drainholes and other such 'traversable wormholes' demand the 'wrong coupling' for their existence. 
would provide in and of itself all the density fluctuations needed to initiate the formation of the voids, walls, filaments, and nodes as they are seen today.

\section{H. Protons, neutrons, and WIMPs as drainholes}

The cosmological model developed in Secs. IV and $\mathrm{V}$ and fitted to the SNe Ia data rested on the assumption that the active gravitational mass densities of primordial matter and continuously created matter are on balance negative. The model did not include (or need to include) a mechanism for producing those imbalances. Subsequently, in Sec. VIB. I proposed as a mechanism that every elementary particle that gravitates is at its core a physical drainhole tunnel, and that the excesses of repulsion over attraction of these tunnels cumulatively produce the overall density imbalances. Among the particles in that category would presumably be counted protons, neutrons, and the weakly interacting massive particles (WIMPs) thought to be the constituents of dark matter. From my perspective the drainhole proposal is simply to modify the conception of every such particle as an entity built around a physical blackhole, by substituting for the blackhole a physical drainhole tunnel. Such a substitution seems to me no more radical than replacing a blocked drainpipe of a wash basin with a drainpipe that has no blockage. But one must in the first place recognize that the basin has a drainpipe, whether blocked or unblocked. I find it more than a little peculiar that there is discussion about the possibility of the Large Hadron Collider's producing a microscopic blackhole through a collision of two protons, when a straightforward application of Einstein's unvarnished theory of gravity suggests that every proton and every neutron in the collider is already built around a blackhole or a collection of blackholes. Are not gravitating bodies just conglomerates of gravitating particles, each making its own small contribution to the gravity of the whole? And if a particle that gravitates has no gravitational sink such as a blackhole or a drainhole associated with it, then what is the source of its gravity?

\section{Galactic nuclei as drainholes}

If instead of a blackhole at the center of our galaxy there is a drainhole, what might it look like? From afar the behavior of matter and radiation at some distance outside such a drainhole would differ very little in appearance from the behavior of matter and radiation at the same distance from the event horizon of a blackhole of the same active mass. Closer in, absence of a horizon in the drainhole makes a big difference. For both a Schwarzschild blackhole of mass $m$ and a drainhole of mass $m$ the radial equation of motion of a test particle is

$$
\frac{d^{2} \rho}{d \tau^{2}}=-\frac{c^{2} m}{r^{2}(\rho)}+(\rho-3 m)\left(\frac{d \Omega}{d \tau}\right)^{2},
$$

with $r(\rho)$ given by Eq. (62) for the drainhole and $r(\rho)=\rho$ for the blackhole. In both cases $\tau$ is the proper time of the test particle. Equation (64) shows that for the drainhole as well as for the blackhole $3 m$ is the radius of its 'capture sphere', as any test particle whose orbit takes it from above $\rho=3 m$ to $\rho=3 \mathrm{~m}$ or below will never again have $d \rho / d \tau>0$, and likewise for photons, $\tau$ being in that case any affine parameter and the first term on the right being absent. Note, however, that upward moving test particles can with sufficient radial velocity rise above $\rho=3 m$ and remain there, and that upward moving photons with angular velocity not too large can escape to $\rho=\infty$, so luminous objects falling into the drainhole and out the far side can in principle be watched from above forever 12

The metric of every cross section of constant $t$ being $d \rho^{2}+r^{2}(\rho) d \Omega^{2}$, the cross sections of the blackhole are euclidean, so the geodesic radius $3 m$ of the capture sphere is also its areal radius. For the drainhole the situation is different: the areal radii $r(2 m)$ of the threshold sphere and $r(3 m)$ of the capture sphere can be made as large as you like by increasing the size parameter $n$, while leaving the mass parameter $m$ unchanged, inasmuch as $m<n<r(2 m)<r(3 m)$, whatever values $m$ and $n$ have.

An analysis in [17] of tidal gradients in the drainhole finds that an object free-falling downward encounters a maximum stretching at $\rho=2 m+n / \sqrt{3}$ (above the hole) and a maximum compression (antistretching) at $\rho=$ $2 m-n / \sqrt{3}$ (below the hole). For a fixed $m$ these have limits proportional to $c^{2} / m^{2}$ as $n / m \rightarrow 1$, and decrease monotonically to 0 as $n / m \rightarrow \infty$. With $m=m_{\mathrm{gc}} \approx 4.31 \times 10^{6} M_{\odot}=6.36 \times 10^{6} \mathrm{~km}$ in geometric units (the current best estimate of the supermass at the center of our galaxy [45]), a star the size of our Sun falling radially downward would experience a maximum stretching difference of accelerations between the leading edge and the trailing edge

12 A complete descriptive catalog of the geodesics of drainholes can be found in Ref. [1]. 
of $56 \mathrm{~km} \mathrm{~s}^{-2}$ if $n=2 m_{\mathrm{gc}}$ and a difference of $8 \mathrm{~km} \mathrm{~s}^{-2}$ if $n=5 m_{\mathrm{gc}}$. The corresponding numbers for the maximum compressing differences are $115 \mathrm{~km} \mathrm{~s}^{-2}$ and $11 \mathrm{~km} \mathrm{~s}^{-2}$. These are small enough that the star would likely survive the journey and show up intact in the region the drainhole exits to on its downside. The same would be true for gas and dust clouds. This is in stark contrast to the supermassive blackhole scenario, in which every bit of matter that falls in encounters unbounded tidal stretching that strips it of its identity as it approaches and reaches the singularity at $r=0$, never to be seen again.

If supermassive drainholes at galactic centers are commonplace, and their exits reside in our (part of the) universe, where should we expect to see those exits, and how might we identify them? In accordance with the notion that the exits of drainhole tunnels tend to isolate themselves by pushing all other matter away, the obvious places to search would be the great voids created by the exits of both the P-matter and the C-matter tunnels. Presumably they would appear as objects of the same sizes as their entrances, emitting pure light intrinsically blueshifted from its journey downward in the drainholes' gravitational potentials, as well as clouds of outflowing gas (and perhaps fleeing stars). The brightnesses of such objects would depend on the rate at which the gases and light were sucked into the drainhole entrances. There being no reports of any such bright objects observed in the voids, one would appear to be left with the alternative that they are there but are too dim to be seen. There is, however, another possibility that seems to me at least as likely.

If, as I proposed in Sec. VIB, every elementary particle that gravitates is at its core a physical drainhole tunnel, then gravitationally every gas cloud or star is simply a conglomeration of entrances of tiny tunnels whose exits are spread throughout the bubble voids of our universe. Each of these little tunnels would be taking in a commensurably small portion of the gravitational ether and pushing it out in a void, there to do its part in causing the bubble (and therefore the universe) to expand. A neutron star would be gravitationally a sieve-like, very dense collection of tiny tunnel entrances; supernova cores would collapse gravitationally to superdense such collections rather than to blackholes. At the center of our Milky Way galaxy there could be then, not a supermassive drainhole, but a supermassive star, formed from the melding of multitudes of gas clouds and stars brought together by their mutual gravitational attractions, comprising gravitationally $4.31 \times 10^{6}$ as many of those tiny drainhole tunnel entrances as are in our Sun - a 'supersun' or 'superstar', so to speak.

There is yet another possibility worth considering. With a little topological imagination one can see how a supermassive drainhole might arise from a plenitude of small dark C-matter drainhole tunnels with their entrances packed tightly together by their mutual attractions. Begin with a pair of them, $T_{1}$ and $T_{2}$ say. If not only their entrances but also their exits were close together, then the coalescing of $T_{1}$ and $T_{2}$ into a single tunnel might be feasible. If, however, their exits had receded far from one another, then the only way $T_{1}$ and $T_{2}$ might reasonably coalesce into one tunnel would be to join at their entrances and close off into a single tunnel connecting their original exits. If that occurred, then the 'gravitational ether' flowing into their entrances would be diverted to the nearby entrances of one or more tunnels, thereby increasing their masses, both the attractive and the repulsive. The remnant of $T_{1}$ and $T_{2}$, no longer connected to the ether flow, would become a gravityless tunnel connecting two distant places out in the voids (perhaps, as suggested in Sec. 7.6, manifesting as a neutrino - or a pair of neutrinos). After many repetitions of this process there would be left a single tunnel large enough to accommodate all the combined ether flow of the C-matter tunnels - a supermassive, dark drainhole. To account for the exit of such a drainhole's being too dim to be observed in the voids, one would have to presume that little if any of the matter in its accretion disk 'accretes' down the drainhole, the majority of it instead being converted into polar jets by one or another of the mechanisms proposed for generation of blackhole jets [46], adapted for drainholes.

\section{SUMMARY}

Exploring the consequences of denying Einstein's 1916 assumption that inertial mass and energy are sources of gravity brings one to the variational principle

$$
\delta \int\left[\boldsymbol{R}-\frac{8 \pi \kappa}{c^{2}}(\mu+\bar{\mu})+2 \phi^{\cdot \gamma} \phi_{\cdot \gamma}-2 \psi^{\cdot \gamma} \psi_{\cdot \gamma}\right]|g|^{\frac{1}{2}} d^{4} x=0,
$$

in which $\phi$ and $\psi$ are scalar fields and $\mu$ and $\bar{\mu}$ are the active gravitational mass densities of distributions of gravitationally attractive and gravitationally repulsive matter, and in which, to accord with the precept that in a space-time manifold nothing extraneous to the metric should participate in the extremizing of the action, only the space-time metric is varied in deriving the field equations to govern the space-time geometry. This logically consistent, purely geometrical version of Einstein's theory of gravity is then found to be capable of performing feats the original version could not perform. Specifically, the modified version is found to be able to:

- Produce cosmological models that replace the 'big bang' with a 'big bounce', include in their expansion inflation, 
deceleration, coasting, and ultimate exponential acceleration, and provide good fits to Hubble plots of type Ia supernovae data.

- Solve the 'Cosmological Constant Problem', by identifying $-\frac{c^{2}}{4 \pi \kappa} \Lambda$ as the net active mass density of gravitating matter.

- Replace the Schwarzschild blackhole with a singularity-free, horizonless, topological 'drainhole' that gravitationally attracts matter on its high, front side while gravitationally repelling matter more forcefully on its low, back side.

- Represent dark matter by the attracting entrance portals of drainhole tunnels and dark 'energy' by the repelling exit portals of those tunnels.

- Suggest a new, drainhole-based mechanism for the creation and evolution of cosmic voids, walls, filaments, and nodes.

- Exorcise 'phantoms', 'ghosts', and 'exotic matter' from the body of gravitational physics.

In view of these successes, as well as its logical consistency, one can justifiably consider Einstein's theory of gravity modified in this way to be an improved version of the original.

[1] H. G. Ellis, Ether flow through a drainhole: A particle model in general relativity, J. Math. Phys. 14 (1973), 104-18; Errata: 15 (1974), 520; see also Sec. VIA of the present paper.

[2] K. A. Bronnikov, Scalar-tensor theory and scalar charge, Acta Phys. Pol. B4 (1973), 251-66.

[3] O. Bergmann and R. Leipnik, Space-time structure of a static spherically symmetric scalar field, Phys. Rev. 107 (1957), 1157-61.

[4] A. Einstein, Essays in Science, (Philosophical Library, New York, 1934) pp. 98-111.

[5] A. Einstein, Die Grundlage der allgemeinen Relativitätstheorie, Ann. der Physik 49 (1916), 769-822, translated in The Principle of Relativity (Dover, New York, 1952) pp. 109-64.

[6] G. Galilei, Dialogues Concerning Two New Sciences (Prometheus Books, Buffalo, N.Y., 1991) pp. 63 et seq.; http://galileoandeinstein.physics.virginia.edu/tns_draft/index.html (pp. 63 et seq.).

[7] A. Einstein, Relativity, The Special and the General Theory: A Popular Exposition, (Methuen, London, 1916), (Bonanza Books, New York, 1961) p. 66; also available as an eBook (Routledge, London, New York, 2002).

[8] H. G. Ellis, Einstein's Real "Biggest Blunder", Int. J. Mod. Phys. 21 (2012), 1242022.

[9] D. F. Bartlett and D. Van Buren, Equivalence of active and passive gravitational mass using the moon, Phys. Rev. Lett. 57 (1986), 21-4.

[10] L. B. Kreuzer, Experimental measurement of the equivalence of active and passive gravitational mass, Phys. Rev. 169 (1968), 1007-12.

[11] C. S. Unnikrishnan and G. T. Gillies, Do leptons generate gravity? First laboratory constraints obtained from some G experiments and possibility of a new decisive constraint, Phys. Lett. A 288 (2001), 161-6.

[12] H. G. Ellis, Leptons might not generate gravity, gr-qc/0308082v3 (2004); NB second sentence of third footnote might need revising.

[13] H. Bondi, Negative mass in general relativity, Rev. Mod. Phys. 29 (1957), 423-8.

[14] D. Hilbert, Die Grundlagen der Physik (Erste Mitteilung), Nachr. Königl. Gesellschaft d. Wiss. Göttingen, Math.-Phys. Kl., 395-407; reprised in Math. Annalen 92 (1924), 1-32.

[15] A. Riess, et al., New Hubble Space Telescope discoveries of Type Ia supernovae at $z>1$ : Narrowing constraints on the early behavior of dark energy, Astrophys. J. 659 (2007), 98-121.

[16] A. Riess, et al., A 3\% solution: Determination of the Hubble constant with the Hubble Space Telescope and Wide Field Camera 3, Astrophys. J. $\mathbf{7 3 0}$ (2011), 119: 1-18.

[17] H. G. Ellis, Cosmic inflation, deceleration, acceleration, dark matter, and dark 'energy' in one coherent package, gr-qc/0701012v7 (2015).

[18] I. Z. Fisher, Scalar mesostatic field with regard for gravitational effects, Zh. Eksp. Teor. Fiz. 18 (1948) 636-640, gr-qc/9911008v1 (1999).

[19] G. Clément, The Ellis Geometry (Letter to the editor), Am. J. Phys. 57 (1989), 967.

[20] L. Chetouani and G. Clément, Geometrical-optics in the Ellis geometry, Gen. Relativ. Gravit. 16 (1984), 111-19.

[21] G. Clément, Scattering of Klein-Gordon and Maxwell waves by an Ellis geometry, Int. J. Theor. Phys. 23 (1984), 335-50.

[22] S. Kar, D. Sahdev, and B. Bhawal, Scalar waves in a wormhole geometry, Phys. Rev. D 49 (1994), 853-61.

[23] C. Armendáriz-Picón, On a class of stable, traversable Lorentzian wormholes in classical general relativity, Phys. Rev. D 65 (2002), 104010-1-10.

[24] H. Shinkai and S. A. Hayward, Fate of the first traversible wormhole: Black-hole collapse or inflationary expansion, Phys. Rev. D 66 (2002), 044005-1-9. 
[25] V. Perlick, Exact gravitational lens equation in spherically symmetric and static spacetimes, Phys. Rev. D 69 (2004), 064017-1-10.

[26] A. Das and S. Kar, The Ellis wormhole with 'tachyon matter', Class. Quantum Grav. 22 (2005), 3045-53.

[27] K. K. Nandi, Y-Z. Zhang, and A. V. Zakharov, Gravitational lensing by wormholes, Phys. Rev. D 74 (2006), 024020-1-13.

[28] T. Müller, Exact geometric optics in a Morris-Thorne wormhole spacetime, Phys. Rev. D 77 (2008), 044043-1-11.

[29] D. K. Dey and S. Sen, Gravitational lensing by wormholes, Mod. Phys. Lett. A 23 (2008), 953-62.

[30] A. G. Doroshkevich, N. S. Kardashev, D. I. Novikov, and I. D. Novikov, The passage of radiation through a wormhole, Astron. Rep. 52 (2008), 685-91.

[31] T. Matos and D. Nuñez, Rotating scalar field wormhole, Class. Quantum Grav. 23 (2006), 4485-95.

[32] K. K. Nandi, I. Nigmatzyanov, and R. Izmailov, New features of extended wormhole solutions in the scalar field gravity theories, Class. Quantum Grav. 25 (2008), 165020-1-19.

[33] F. Abe, Gravitational microlensing by the ellis wormhole, Astrophys. J. 725 (2010), 787-93.

[34] K. Nakajima and H. Asada, Deflection angle of light in an Ellis wormhole geometry, Phys. Rev. D 85 (2012), 10501-1-3.

[35] A. J. S. Hamilton and J. Lisle, The river model of blackholes, Am. J. Phys. 76 (2008), 519-32.

[36] N. Tsukamoto, T. Harada, and K. Yajima, Can we distinguish between black holes and wormholes by their Einstein ring-systems?, Phys. Rev. D 86 (2012), 104062-1-6.

[37] A. Einstein and N. Rosen, The particle problem in the general theory of relativity, Phys. Rev. 48 (1935), 73-7.

[38] H. G. Ellis, Space-time-time: Five-dimensional Kaluza-Weyl space, gr-qc/0107023 (2001); Space-time-time, gr-qc/0205029 (2002).

[39] H. G. Ellis, Quantum effects from a purely geometrical relativity theory, J. Phys. Conf. Ser. 24 (2005), 225-30, physics/0505139 (2005).

[40] R. G. Vishwakarma and J. V. Narlikar, Modeling repulsive gravity with creation, J. Astrophys. Astr. 28 (2007), 17-27.

[41] J. V. Narlikar and T. Padmanabhan, Creation-field cosmology: A possible solution to singularity, horizon, and flatness problems, Phys. Rev. D. 32 (1985), 1928-34.

[42] H. G. Ellis, Darkholes: Nicer than blackholes — with a bright side, too (Does energy produce gravity?), gr-qc/0003024 (2000).

[43] S. A. Thomas, F. B. Abdalla, and O. Lahav, Upper bound of $0.28 \mathrm{eV}$ on neutrino masses from the largest photometric redshift survey, Phys. Rev. Lett. 105 (2010), 031301-1-4.

[44] P. J. E. Peebles, The void phenomenon, Astrophys. J. 557 (2001), 495-504.

[45] S. Gillessen, et al., Monitoring stellar orbits around the massive black hole in the galactic center, Astrophys. J. 692 (2009), 1075-1109.

[46] M. A. Abramowicz and P. C. Fragile, Foundations of black hole accretion disk theory, Living Rev. Relativity 16 (2013), 1, http://www.livingreview.org/lrr-2013-1. 